ISSN: $1782-2041$

DOI: $10.25518 / 1782-2041.1131$

\title{
Recensions (décembre 2019)
}

Filippo Muratori \& Valeria Bizzari, Alle origini dell'autismo: Il ruolo dimenticato di George Frankl, Roma, Fioriti Editore, 2019, 95 pages. ISBN : 978-8898991907. Prix : $14 €$.

What is a mental disorder? That should probably be the starting point of each paper or review that deals with psychopathology. Since time immemorial, human being has tried to unveil and clarify the core nature of these severe diseases. For instance, Ajax Telamonio, one of the heroes of the Homer's Iliad, did not obtain Achilles' weapons, in favor of Odysseus. Blinded by grief, Ajax decided to kill those responsible for the decision, but he suffered an incantation by the goddess Athena and, after falling asleep, he massacred a flock of sheep. When he woke up, he realized what he had done and, for the shame, he killed himself. Interestingly, such "pantoclastic crisis" - we would say nowadays - was caused by a supernatural entity. A similar example may be the myth of Bellerophon, whose melancholic state was determined by his hybris towards Olympic gods. In other terms, the first, mythological depictions of mental disorders intertwined human nature with godly entities. No mental health without supernatural support.

Of course, the history of mental illness is not the aim of this review. Nevertheless, the abovementioned examples may illustrate a tendency which is today again in vogue. The tendency to attribute non-human aspects to mental diseases. Indeed, the ultra-reductionist theory is the most widespread in the panorama of psychiatry. In this perspective, what we experience in our lifeworld is nothing more than an internal construction. We simply become aware of what happens inside our body throughout the elaborating process of the brain, which finally elaborates the illusion of our existence itself. Our consciousness, according to this view, is a simulation of the interaction between 
the body and the environment and our subjectivity is the illusion created by this simulation. Again, there is something exceeding human nature. Furthermore, there is also no space for the clinical encounter. Since consciousness is a simulation and subjectivity an illusion, it appears worthless to understand patients' way of suffering and to look for different chances of living freely. From this ultra-reductionist point of view, the decisive level is the internal mode of processing and elaborating what is pretended to be life. It is not the constant encounter between me, the others and the world that determines, on the one hand, the way by which I experience my self and, on the other, the way throughout which the elaboration of further experiences becomes possible. But it is the mode of elaborating that establishes the way of encountering the lifeworld. No mental health without biochemical support.

From this perspective, autism spectrum conditions are conceived as $a$ deficit in the capacity of internally elaborating interpersonal experiences. Autism is considered to be a sociality disorder, but sociality is interpreted as a construction of the brain. Paradoxically, social context is unnecessary for the establishment of sociality, just a brain is needed.

Having made these considerations, the intent of the book of Filippo Muratori and Valeria Bizzari appears very important. In fact, they rediscover the work of one of the pioneers of autism research, George Frankl. According to him, the core problem of autistic children is a disturbance of the affective contact. Humans use both affective and verbal language to communicate. Affective language consists of non-verbal communicative symbolizations (for instance, facial expressions, bodily gestures, tone of voice). Verbal language includes all the verbal production used to talk with other people. While nonautistic people can utilize unproblematically affective and verbal language together, autistic people cannot. In this sense, we might say that autism is a communication disorder. To communicate, human being should firstly establish an affective contact with others. And it is on the basis of this contact that communication and language become possible. But "according to Frankl, child autism has its core in the distortion or loss of the affective contact" (p.76). And this psychopathological nucleus allows Frankl to phenomenologically investigate autistic Gestalts: anomalies of the affective and verbal language may be understood on the light of this core psychopathology. Since mental disease is "expression of a specific variation of the being-in-the-world of the affected subject" (p. 77), autism spectrum conditions may be conceived as a differing way of being-in-the-world that reveals itself through particular ways of communication.

Again, according to Frankl, there are two modes of communicating: affective and verbal language. Interestingly, he considered affective contact

\section{2}

Bull. anal. phén. XV 10 (2019)

https://popups.uliege.be/1782-2041/ @ 2019 ULiège BAP 
not only as the psychopathological core of autism, but also as crucial for communicating. Therein, he traces Merleau-Ponty's notion of the constitution of subjectivity as embodied and intersubjective. Affective language is primarily revealed in facial gestures, bodily attitudes, reciprocal glances. And, once a child is born and during the first year of life, it is the principal and crucial mode of being-towards-the-world and of communicating. Furthermore, it is starting from this primary intersubjectivity that "the body becomes the place throughout which shared meanings emerge" (p. 80). Once shared sense-making throughout bodily resonance and primary intersubjective encounter is not at disposal, anomalous modes of communication unfold. In particular, verbal language is altered in artificial ways such as monotone verbal reproduction, automatic language, "scan" language, declamation language.

Finally, we may say, with the authors, that "what is lacerated in the autistic experience is not only a neural substrate, or cognitive faculties, but the dynamic and interpersonal self, usually provided of an implicit relational knowledge, an "affective language", namely an embodied and intuitive intersubjective understanding, which makes him able to interact, to "dance with others", long before verbal communication, or "language of word", is developed" (p.91).

In conclusion, this book of Filippo Muratori and Valeria Bizzari, dealing with George Frankl's interpretation of autism, is of particular importance to understand the essence of a condition, which well represents fundamental alterations of being-towards-the-world and of communicating in a shared sociality. No mental health without affective contact.

Raffaele Vanacore

(Università degli Studi della Campania Luigi Vanvitelli)

Guidi Cusinato, Biosemiotica e psicopatología dell' "ordo amoris ». In dialogo con Max Scheler, Milano, Franco Angeli, 2018, 292 pages. ISBN : 9788891780645. Prix : $33 €$.

Guido Cusinato ist gegenwärtig einer der tiefsten Kenner und Interpreten Max Schelers. Im Zuge der letzten Jahrzehnte hat er nicht nur in seiner Amtszeit als Präsident der Max-Scheler-Gesellschaft einen wichtigen Beitrag zur Überlieferung und Erweiterung der Forschung zu Scheler geleistet, sondern in seinem Euvre eine eigenständige Lesart der Schelerianischen Werke entwickelt. Die vorliegende Arbeit gliedert sich deswegen in den 
Zusammenhang verschiedener vorheriger Arbeiten ein, insbesondere der ins Deutsche übertragenen Arbeit „Person und Selbsttranszendenz. Ekstase und Epoché des Ego als Individuationsprozesse bei Schelling und Scheler“ (2012, Königshausen \& Neumann). Zum Verständnis von „Biosemiotica e psicopatología dell'ordo amoris“ ist vornehmlich die argumentative Kontinuität von Cusinatos philosophischen Reflexionen bedeutsam, ein grundsätzlich neuer Beitrag ist hingegen die Kontaktaufnahme mit Psychiatrie und Psychopathologie.

Der philosophische Grundgedanke des Werkes ist die Autonomie des lebendigen Organismus gegenüber der linearen Naturkausalität. Cusinatos Ansinnen ist, verschiedenen zeitgenössischen Auffassungen, wie dem Emergentismus, den Standpunkt einer in der Natur stufenontologisch manifestierten Freiheit entgegenzustellen. Dabei schließt er an Friedrich Wilhelm Schelling mit der Auffassung an, dass es eine „nicht-mechanische physische Kausalität“ (S. 28) gäbe. Bei Schelling heißt es entsprechend: „Diese Causalität heißt Leben. - Leben ist die Autonomie in der Erscheinung, ist das Schema der Freiheit, insofern sie in der Natur sich offenbart" (Schelling, 1856, 249). Mit Thomas Fuchs denkt Cusinato diese Kausalität als ,vertical and horizontal circular causality“ (Fuchs, 2017, 95) im Sinne von „embodied freedom“, stellt also der Linearität der anorganischen Kausalität eine zirkuläre gegenüber. Maßgeblich ist für Cusinato dabei, den zeitlichen Zusammenhang nicht als Sukzession, sondern als Simultanität in einem geschlossenen System aufzufassen. Für die Erklärung der zirkulären Kausalität nimmt der Autor einen fortschreitenden Selbstbezug auf sich selbst [progressivo ripiegamento autoreferenziale verso sé stesso, 32] und ermöglicht dadurch die Kompatibilität mit den enaktivistischen, aber auch systemtheoretischen Darstellungen der Autopoiesis.

Die eigentliche Wendung der Arbeit betrifft allerdings nicht die Sonderstellung der organischen Kausalität, sondern bezieht sich auf sie, um die dem Organismus eigentümliche Beziehung zum Ausdruck zu charakterisieren. Cusinato sagt: „Im Mechanismus wird die Finalität durch die Außenwelt aufgeprägt [impressa]“ — „Im Organismus hingegen wird die Finalität durch den Organismus selbst in die Außenwelt enaktiv ausgedrückt [espressa]" (S. 34). Die organische bzw. verkörperte Kausalität müsse deswegen ursprünglich als eine expressive Kausalität verstanden werden. Während dieser Schritt gewiss von größter Bedeutung ist und den Begriff des Ausdrucks zurecht in den Mittelpunkt der Betrachtung stellt, so ist allerdings fragwürdig, ob Cusinatos Behauptung zutreffe, dass ,die Aktivität des Organismus nicht direkt gegen die Umwelt, sondern auf die Aufrechterhaltung eines inneren 
Äquilibriums gerichtet“ (S. 36) sei, weswegen „das Vermögen das Äquilibrium in Auseinandersetzung mit der Variabilität der Welt aufrechtzuerhalten der Schlüssel für das Problem der organischen Identität und somit der Philosophie des Organischen" (ebd.) werde. Diese Bestimmung des Organismus durch den Begriff der Selbstregulation hat seinen historischen Vorläufer im Empiriokritizismus und droht, die Bedeutung der Subjektivität zu unterschätzen.

Dessen ungeachtet gelingt es Cusinato, auf Grundlage seiner Annahme einer kausalen Autonomie des Organismus eine gelungene Kritik des (ontologisch-)emergentistischen Konzeptes der „downward causation“ vorzutragen: „Ein Organismus emergiert nicht aus der eigenen Zelle wie ein Tornado aus den Wassertropfen und dem Wind, die in der Atmosphäre sind" — „Ein Organismus ist ein autopoietisches System, ein Tornado nicht“ (S. 43). Der Gedanke ist dabei, dass die Eigenständigkeit und Neuheit des Organismus gegenüber seiner Umwelt nur durch eine „top-down“ oder „upward“ Kausalität — in der die Wirkung ihre Ursache übersteigt [eccede] — erklärt werden kann, deren Funktionsweise Cusinato mit dem Begriff des „Stoffwechsels“ [metabolismo] erklärt: „Der Organismus entsteht vermittels der Verstoffwechselung der Elemente in der Umgebung, während der Tornado umgekehrt ohne das Bedürfnis, die Wassertropfen $\mathrm{zu}$ verstoffwechseln, emergieren kann, nämlich schlechterdings als Resultat von deren Aggregation" (S. 44).

Es ist der Schlüsselbegriff des Stoffwechsels resp. Metabolismus, mit dem Cusinato die Bedeutung der lebendigen Erfahrung für die Kausalität des Organismus aufzeigt, also der durch den Begriff ,Adaptivität ' bei Varela bezeichneten Verbindung zwischen Autopoiesis und ,sense making“. Weil die Untersuchung der Adaptivität bereits auf bakteriellem Niveau ergebe, dass „ein System beim Anpassen an eine Umwelt nicht nur auf das Überleben achtet, sondern auch eine eigene Form von Interessen und eine eigene Präferenzordnung ausdrücken kann" (ebd.), wird zu ihrem Verständnis die primordiale Wertkartierung [mappatura valoriale primordiale] salient. Die Funktionsweise, mit der die „upward causation“ des Stoffwechsels den Organismus konstituiert, verweist also auf eine Sphäre der ,primordial affectivity“, die Cusinato mit Begriffen wie Triebstruktur, Leibschema und Gefühlsdrang thematisiert. Der genuin Schelerianische Beitrag ist unterdessen in der Wertorientierung gegeben und insbesondere im Begriff der „Wertnehmung“. Der Autor behauptet deswegen, „dass das Körperschema, die Wertnehmung und die Interaktion mit dem Ausdrucksfeld des Lebens imstande sind, einer Form des ,Stoffwechsels von Ausdrücken“ Leben zu schenken, nämlich einer Form von affektivem Stoffwechsel auf zweiter Ebene in biochemischer Hinsicht“" (S. 46). 
Im Mittelpunkt dieser Überlegungen steht der Begriff des Ausdrucks, den Cusinato biosemiotisch denkt und bereits auf dem Niveau der Biosynthese realisiert sieht. Der Ansatz der Biosemiotik gestattet es jedoch zugleich, den Phänomenkreis des Psychischen zu integrieren und dementsprechend eine Leib-Seele-Fissur zu vermeiden: „Psychisch, im Gegensatz zum Unbeseelten, ist all' das, was ein Innesein vom Standpunkt des Für-sich-Seins ausdrückt" (S. 51). Dieser Verweis auf das Innesein ist dabei gerade nicht als eine Vorgegebenheit gegenüber dem Ausdruck zu lesen, sondern als eine „lebendige Einheit von Erlebnis und Ausdruck" (S. 107). Demnach vertritt Cusinato keinen naiven Immanentismus oder Spiritualismus, ohne jedoch die eigenständige Phänomenalität des Psychischen zu leugnen. Hierin liegt die eigentümliche Stärke der Stufenontologie Schelers und Hartmanns: im Gegensatz zu Emergentismus, der die Natur der niederen Schichten unberührt lässt, deren Transformation anzunehmen - das Verhalten einer Zelle, die am affektiven Metabolismus teilhat, kann nicht aufgrund ihrer anorganischen Komponenten vorhergesagt werden.

Den Gehalt der Biosemiotik begreift der Autor unter Bezugnahme auf die Wertphilosophie Schelers: „die lebendige Relevanz [rilevanza vitale] richtet die Sinnesorgane zu einer Erkundungshandlung aus, mit dem Zweck, eine semiotisch-werthafte Karte der Gegenstände in der eigenen Umwelt zu zeichnen" (S. 66). In diesem Gedanken spiegelt sich die emotionalistische Überzeugung Schelers wider: „Die Wertnehmung geht stets der Wahrnehmung vorher" (Scheler, 1926, 122). In anderen Worten: Das eigenständige Verhalten des Organismus setzt eine Ausrichtung seiner Bedürfnisse und Interessen an der Werthaftigkeit bzw. Relevanz seiner Umgebung für ihn voraus. Diese Vermittlung fasst Cusinato einerseits leibphänomenologisch mit dem Begriff des „Leibschemas“ andererseits semiotisch mit dem Hinweis auf die Beziehung zwischen Scheler und Uexkülls Vorstellung von „Merkwelt“ und „Funktionskreis“ auf.

Dementsprechend wendet sich der Autor im dritten Kapitel seiner Arbeit der Bestimmung des Leibschemas aus Schelerianischer Perspektive zu: „Die wahrhaftige Neuigkeit entsteht, wenn Scheler in ,Erkenntnis und Arbeit erklärt, dass das Lebensgefühl tatsächlich die Gefahren und Gelegenheiten allein auf Grundlage einer eigenen, ursprünglichen Aktivität der Fantasie antizipieren kann, d. h. unabhängig vom Intellekt: die Triebfantasie" (S. 95). Die Triebfantasie ergebe sich dabei aus der spezifischen Intentionalität und dem Tatendrang [progettualità] des Subjekts. Allerdings sei sie nicht als eine Konstruktion oder Produktion misszuverstehen, sondern als Partizipation am 
biosemiotischen Prozess vermittels einer „Grammatik des Ausdrucks“ (Scheler, 1973, 92). Der Organismus kann sich in seiner Triebfantasie also einer im Ausdrucksfeld angelegten Kreativität bedienen, der seine Merkwelt entspricht.

Vor diesem Hintergrund wird deutlich, dass Cusinato auch die Leiblichkeit durch die Phänomenologie des Ausdrucks zu begreifen versucht. Weil im vierten Kapitel die „direkte Wahrnehmung des Ausdrucks“ (S. 101) besprochen wird, handelt es sich um das Schlüsselkapitel für das Verständnis von Cusinatos Ansatz. Weil der Begriff des Ausdrucks sozial- und kommunikationswissenschaftlich usurpiert ist, mag sich in Anbetracht von Cusinatos Auffassung, dass bereits auf mikrobiologischem Niveau von Biosemiotik des Ausdrucks gesprochen werden kann, ein Anthropomorphismus-Verdacht aufdrängen.

Diesem Verdacht lässt sich Cusinatos Begriff der ,,unipathischen Affektivität" [affettività unipatica] entgegenhalten, wobei das Unipathische aus Schelers Emotionsphänomenologie der ,Einsfühlung' abgeleitet ist. Dementsprechend kennzeichnet der Autor zwei Aspekte der unipathischen Affektivität, einerseits den oftmals als „direkte Empathie“ oder „direkte Wahrnehmung" beschriebenen Mitvollzug fremdpsychischer Akte, der im fünften Kapitel diskutiert wird. Andererseits die diese Form der Empathie metaphysisch noch fundierende Idee, „die die Einsfühlung [unipatia] mit der ursprünglichen Form allen emotionalen Nachempfindens [emotional sharing] identifiziert, welche unter der Herrschaft einer gemeinsamen ,Grammatik des Ausdrucks" des Alllebens stehe" (S. 115). Der Ausdruck wird somit als metaphysisches Fundament der Darstellung aufgefasst, als „der lebendige Zustand der Materie" (S. 102).

Angesichts dieser grundlegenden Funktion des Ausdrucksbegriffes scheidet der Autor die Expressivität, welche auch dem Anorganischen, etwa einer Landschaft, zukommen kann, vom Ausdruck im engeren Sinne, der als intentional verstanden werden muss: „Der Organismus ist das Ausdrucksfeld, das sich um das Bedürfnis, die eigene gefühlte Existenz auszudrücken, herum konstituiert und die Morphologie des Lebens ist die Form seiner expressiven Bewegung" (S. 103). Mit Gedanken wie diesem ist der Anspruch eines das ganze Leben umfassenden Ausdrucks etabliert. Die weiteren Überlegungen widmen sich hingegen der Versöhnung dieser metaphysischen Grundsätzlichkeit mit den unmittelbaren Phänomenen des Ausdrucks.

Das maßgebliche Feld für das Erlebnis des Ausdrucks ist die Intersubjektivität, wobei unter diesem Begriff gerade nicht eine Kommunikation zwischen zwei gänzlich unabhängigen Monaden zu verstehen ist. Cusinato liest Schelers Alteritätsphilosophie durch Autoren wie Kimura und Buber zugunsten einer radikalen Priorität der Mitwelt vor der Innenwelt, sodass „die 
von der schwarzen Sonne der Jemeinigkeit ausgehende Gravitationskraft überwunden und die inter-subjektive Beziehung in eine anthropogenetische Beziehung der Inter-Formativität [inter-formatività] umgestürzt wird“ (S. 201). Dabei geht der Autor aber nicht so weit, eine vollständige Transparenz des anderen und somit die Aufhebung der singulären Subjektivität anzunehmen (vgl. S. 113). Allerdings nehmen einige Behauptungen synechologische Züge an und drohen, wie zuvor bereits die Vorstellung eines Äquilibriums sowie an anderer Stelle die Auffassung des Subjekts als „Rezeptionszentrum“ (vgl. S. 200ff), die Bedeutung der Subjektivität zugunsten kosmovitaler Dynamiken zu vernachlässigen.

Im Anschluss an diese Behauptungen kommt Cusinato dazu, $\mathrm{zu}$ zeitgenössischen Kontroversen der Phänomenologie Stellung zu nehmen. So kommentiert er beispielsweise die Arbeit von Fuchs und De Jaegher zu „unidirectional incorporation“ und „mutual incorporation“ mit dem Vorschlag, anstelle einer kontinuierlichen intersubjektiven Synchronisation von Leibschemata anzunehmen, dass vielmehr bereits ursprünglich eine unipathische Synchronisation vorliege, welche die „mutual incorporation“ allererst ermögliche. In diesem Sinne liest er Fuchs' Begriff der ,primären Empathie' und hebt hervor, dass die Beziehung zum Leibschema des Anderen nur vermittels der Wertnehmung und der prärepräsentativen Dekodierung der Grammatik des Ausdrucks möglich wäre.

Cusinatos Vorstellung von der Inter-Formativität findet in der Allegorie der ,Wasserspiele eines tanzenden Springbrunnens“ ihren Ausdruck: „der Spritzer, der aus dem Springbrunnen sprudelt, nimmt im Fluss des Wassers, gemäß der Position und Form der Düsen, von denen der Austritt bestimmt wird, eine ,individuelle‘ Form an. Der Spritzer ist kein Daseiendes, das sich allein in Bezug auf sich selbst konstituiert, sondern im beständigen Bezug auf die Wasserleitung“" (S. 154). Die Abstimmung zwischen zwei Tropfen bzw. Subjekten ergebe sich folglich nicht aus zwischenleiblicher Resonanz, sondern aus dem gemeinsamen Ursprung in den Düsen bzw. im Lebensdrang. Die Ausprägung dieses Lebensdranges werde indessen von allen Lebewesen auf ihre Weise gemäß der gemeinsamen Grammatik des Ausdrucks bestimmt. Mit Lebensdrang, einer auch Bergsons Denken verwandten Wendung, schlägt Cusinato eine metaphysische Brücke von Scheler zum Begriff des ,conatus bei Spinoza, den Fuchs für die Psychopathologie fruchtbar gemacht hat.

Diese Überlegungen zur intersubjektiven bzw. interformativen Dynamik der primordialen Affektivität überträgt Cusinato im zweiten Teil des Werkes auf die Psychopathologie. Der Autor betont dabei, dass es zwischen Scheler und namhaften phänomenologischen Psychiatern wie Jaspers deutliche Bezüge gebe. So gelte beispielsweise die Auffassung des Symptoms als

\section{8}

Bull. anal. phén. XV 10 (2019)

https://popups.uliege.be/1782-2041/ @ 2019 ULiège BAP 
nicht auf eine objektive Gegebenheit Reduzierbares sowohl für Jaspers als auch für Scheler. Ferner habe Schelers Werk einen maßgeblichen Einfluss auf Binswanger, Gebsattel, Minkowski, Straus und Schneider gehabt, weswegen eine Auseinandersetzung mit Scheler zur Erweiterung der Psychiatrie nicht erst ein aktuelles Anliegen ist.

In jüngster Zeit sei es allerdings John Cutting gewesen, der Scheler für die Psychiatrie fruchtbar zu machen versucht hat. Entscheidend sei dabei Schelers im Aufsatz ,Über Selbsttäuschungen“ von 1912 formulierte Vorstellung, dass „die psychopathologischen Störungen als Zerstückelung der lebendigen Wirklichkeit in mechanische Momente beschrieben werden können" (S. 175). So zeige sich, dass erst die betroffenen Patienten, nicht aber gesunde Menschen eine explizite Zuwendung auf diejenigen Phänomene vorwiesen, die normalerweise präreflexiv sind. Deswegen könne beispielsweise die Amnesie als „Hyperreflexivität“ (S. 174) beschrieben werden, insofern als die Personen ihre natürliche Orientierung verloren hätten und die einst selbstverständlichen Erfahrungen gleichsam mechanisch und körperlich statt leiblich erfahren würden, weswegen bereits bei Scheler die Diagnostik des „disembodiment“" angelegt sei.

Für das komplexe Störungsbild der Schizophrenie sei hingegen, und dabei greift Cusinato auf Cutting zurück, eine ausführlichere phänomenologische Analyse erforderlich. So sei es die Wertnehmung, die bei Schizophrenen gestört sei: „Die Störung der Wertnehmung unterbricht den anthropogenetischen Strukturprozess, der an der Basis der menschlichen Existenz steht. Am Ursprung gibt es eine Transformation der Wertnehmung in eine sterilisierte und nur Teilweise mit der Wertschicht der Wirklichkeit und der Ausdrucksschicht des Lebens verknüpfte Wahrnehmung“ (S. 187). Somit kommt Cusinato zum Urteil, dass es zwar sein mag, dass sich ein schizophrener Patient beispielsweise für religiöse Werte interessierte, sie doch aber nicht im eigentlichen Sinne, verstoffwechselte' [metabolizza], d. h. dank seines ordo amoris mit dem Rest der Welt synchronisiere.

Mit dem Begriff des ordo amoris, der im Mittelpunkt von Schelers Wert- und Liebesphänomenologie steht, gewinnt Cusinato den Kernbegriff seiner psychopathologischen Überlegungen. Dabei handelt es sich in der Normalform um die individuelle Vorzugsordnung, die die Voraussetzung für das Fühlen der Werte als Fundament der Wahrnehmung sei. Zugleich ist der ordo amoris neben dem ,Aktzentrum' einer der Schlüsselbegriff für Schelers Personalismus, weswegen Cusinato von ihm als ,principium individuationis der Person“ (S. 192) spricht. Eine Störung des ordo amoris erfolge beispielsweise durch das Ressentiment, bei dem die Abstimmung (sintonizzazione) mit 
der Welt misslänge. Im Falle des Schizophrenen hingegen gehe die Entfremdung des ordo amoris bis zum Kontaktverlust mit der Ausdrucksschicht des Lebens, was ihn letztlich auf sich selbst in einer Art Egozentrismus zurückwerfe. Neben der schizophrenen gäbe es in Hinsicht auf den ordo amoris eine zweite grundsätzliche Störungsform: „Wenn der Schizophrene dazu tendiert sich selbst und die Anderen wie Roboter zu sehen, tendiert der Melancholiker dazu, sich selbst und die Anderen auf die soziale Rolle und das soziale Selbst zu reduzieren“" (S. 223).

Es kann nicht unbemerkt bleiben, dass Cusinatos Reflexionen auf die Psychopathologie nicht weit aus der Warte des Philosophen hinaustreten. Seine Überlegungen bleiben zumeist fundamental und sind nicht erkenntlich vom hippokratischen Drang nach Therapie motiviert. Bis zu einer Schelerianischen Psychiatrie oder Psychotherapie bedarf es also noch einiger Zwischenschritte und Auseinandersetzungen. Das ist allerdings auch dem Umstand geschuldet, dass Cusinatos Anspruch groß ist und sich mit Gewissenhaftigkeit aus seinen langjährigen philosophischen Erörterungen entwickelt.

Insgesamt handelt es sich bei „Biosemiotica e psicopatología dell'ordo amoris" um die Pionierleistung eines Brückenschlags zwischen Schelerianischer Philosophie und Psychodiagnostik. Dass dabei noch nicht alle Fragen beantwortet werden können, sondern vielmehr zunächst Problemfelder gesichtet werden, liegt in der Natur der Sache. Entscheidend ist einerseits die Philosophie des Ausdrucks als ein tiefes und bedeutungsschwangeres Feld, dessen Erkundung Cusinato mit der vorliegenden Arbeit im Kontext der wichtigen zeitgenössischen Fragen vorangetrieben hat. Andererseits bestimmt er die Eigenständigkeit emotionaler Phänomene und den Begriff des Wertes im Sinne des „emotional turn“ (Fischer, 2015) als Desiderata der Psychologie, Psychopathologie und Psychiatrie.

\section{Literatur}

Fischer, J. (2015). Der Emotional Turn in den Kultur- und Sozialwissenschaften aus der Perspektive Max Schelers. Thaumàzein, 3, 11-28.

Fuchs, T. (2017). Ecology of the Brain. Oxford: Oxford University Press.

Scheler, M. (1926). Die Wissensformen und die Gesellschaft. Leipzig: Der Neue-Geist Verlag.

Scheler, M. (1973). Wesen und Formen der Sympathie. Bern: Francke.

Schelling, F. W. (1856-1861). Sämtliche Werke in 14 Bänden. Stuttgart und Augsburg: Cottascher Verlag.

Alexander Nicolai Wendt

(Universität Heidelberg) 
Thomas Fuchs, Ecology of the Brain. The Phenomenology and Biology of the Embodied Mind, Oxford University Press, 2018, 368 pages. ISBN : 9780199646883. Prix : $42 €$.

Thomas Fuchs, psychiatre et professeur de philosophie de l'Université de Heidelberg, reconnu internationalement pour ses travaux dans le domaine de la psychopathologie phénoménologique, expose dans son dernier ouvrage une pensée à la croisée des chemins. En effet, Fuchs fait jouer de concert la phénoménologie, la biologie, l'éthologie et les neurosciences afin de proposer une approche écologique du cerveau. Fuchs déconstruit avec minutie les thèses réductionnistes du paradigme neuroconstructiviste selon lequel la subjectivité humaine ne serait qu'un corrélat de processus neuronaux. À l'encontre de cette conception, Fuchs présente un concept spécifique de la vie où le cerveau n'est plus déterminé comme le producteur de la vie mentale, mais comme un organe de médiation. En tant que tel, lui est imputé la fonction de médiatiser tant les processus inhérents à l'organisme que celui-ci avec son environnement et avec autrui. Le cerveau est dès lors à envisager, non pas comme un organe de traitement de l'information, mais comme un organe de transformation. Voilà pour la trame générale de l'ouvrage.

Fuchs commence par critiquer le fer de lance de la neurologie, à savoir la tentative de réduction de la sphère subjective à la matière physiologique cérébrale. Fuchs rapporte notamment les propos de Thomas Metzinger, qui déclare : « Nous sommes des Ego-Machines, mais nous n'avons pas de Soi. Nous ne pouvons pas quitter le tunnel de l'Ego parce qu'il n'y a personne qui pourrait en sortir. [...] En fin de compte, l'expérience subjective est un formatage de données biologiques, un mode hautement spécifique de présentation d'informations sur le monde, et l'Ego s'avère simplement un événement physique complexe - un modèle d'activation dans votre système nerveux central »(p. 31). L'expérience, tant du monde que de notre propre subjectivité, s'avère une construction produite par notre cerveau.

En guise de conséquence, notre rapport au monde ne peut être immédiat, il ne nous donne pas accès à la chose même, mais de manière médiate au moyen de représentations mentales produites par notre cerveau. Ainsi, le matérialisme réductionniste des neurosciences s'accommode fort bien d'une épistémologie représentationaliste d'après laquelle l'accès au monde externe ne nous est autorisé qu'en vertu de représentations issues du cerveau et que nous avons de ce monde. La doxa fait la part belle à cette épistémologie, puisque la neuroimagerie nous fournirait la preuve ontologique de cette idée. 
Et de fait, la technologie, semble-t-il, nous permet aujourd'hui d'observer, en direct, notre cerveau en train de penser.

Le cœur de la critique avancée par Fuchs trouve son fondement dans une réforme radicale consistant à intégrer à une phénoménologie du corps propre une fondation biologique de l'organisme vivant. Point de départ de son modèle écologique, Fuchs décrit l'organisme comme une unité subjective, incarnée, et en interaction constante avec son environnement, de laquelle interaction émerge un monde de significations spécifiques, une Umwelt, pour ce même organisme (Fuchs s'inspire notamment des travaux de von Uexküll). Cette amorce est suivie de conséquences en cascade. Parmi celles-ci, et d'une part, nous ne percevons pas le monde au moyen de représentations, mais toujours en regard de notre position corporelle située dans ce monde. D'emblée, les objets expriment pour nous diverses possibilités de nous rapporter à eux, ils nous ouvrent à une potentialité d'actions. D'autre part, outre la phénoménologie d'obédience merleau-pontienne, Fuchs s'inspire de l'approche énactive de la cognition (Francisco Varela, Alva Noë, etc.) selon laquelle la réalité n'est pas quelque chose d'extérieur et de prédéterminé, mais est continuellement amenée à l'existence par l'interaction sensori-motrice de l'organisme dans son environnement.

À ce stade, Fuchs avance une thèse centrale et qui cherche à éviter l'écueil du dualisme corps-esprit qui prévaut encore dans la Philosophy of mind. En partant de l'unité de l'organisme vivant, deux points de vue à propos de celui-ci se dessinent. D'un côté, l'organisme se vit lui-même comme corps vivant, comme Leib. D'autre part, il se donne également comme corps physiologique, comme Körper. Cependant, Fuchs n'édifie guère un nouveau dualisme. Il défend un «monisme médiatisé » ou, s'inspirant explicitement de la terminologie de Hegel, une unité dialectique de l'unité et de la diversité (p. 80). Ces deux modalités du corps résonnent au sein de la même personne. À titre d'exemple, lorsque sa main est piquée par l'aiguille du médecin, la douleur éprouvée par le patient est située là où le corps physique se trouve percé. Il n'y a pas, d'un côté, la main vécue et, de l'autre, la main physique, mais une seule et unique main qui se donne comme une "unité physicoesthésiologique », comme l'affirmait Husserl. Est donc présente une «coextensivité », ou une "syntopie », du corps vécu et du corps organique au sein de la même personne.

Par ailleurs, et se revendiquant de Plessner, cette ontologie du corps est corrélée à une approche originale de la subjectivité que Fuchs nomme «la double aspectivité de la personne » et qui caractérise l'étude écologique de la vie. Le premier aspect est « l'attitude personnaliste » qui désigne le discours que la personne exprime à propos de ses propres énactions vitales, dans sa 
rencontre immédiate avec autrui. Le second aspect est rendu par « l'attitude naturaliste » qui constitue la perspective de l'objectivation du corps où celuici fait l'objet de diverses explications physiologiques. Pour autant, cette attitude ne s'excepte jamais du monde de la vie :

Même si nous pouvons nous approprier cette seconde attitude naturaliste, la primauté de la perspective en première et en seconde personne, ou la perspective du participant, reste toujours vraie. Si nous n'étions pas toujours en mesure de percevoir et d'interagir avec les autres en tant qu'êtres de notre propre genre, c'est-à-dire comme sujets incarnés, nous ne serions pas même capables d'identifier les phénomènes dont nous cherchons à trouver les corrélats neuronaux ou physiologiques. [...] En aucun cas les phénomènes de la conscience ne peuvent être étudiés en faisant simplement varier les stimuli environnementaux et en mesurant des activités neuronales correspondantes (p. 213-214).

Mais qu'en est-il précisément du rôle du cerveau dans le cadre de cette écologie de la subjectivité incarnée ? Pour quelles raisons est-il à la fois un organe de médiation et de transformation? Une myriade de concepts fait l'objet d'une attention particulière. En premier lieu, la vie et le corps vécu constituent la source fondamentale de notre énaction, laquelle inclut les activités de la conscience, tant réflexives que pré-réflexives. La vie n'est donc ni le fait d'une conscience pure ni ne se réduit à un organisme physique. La vie couvre l'ensemble de nos manifestations corporelles et subjectives. En second lieu, les organismes vivants sont des corps physiques complexes, des systèmes, qui, suivant le fonctionnement continuel de leur métabolisme, maintiennent leur forme et leur structure dans le temps. L'organisme vivant est donc un système auto-organisé (ou autopoiétique) en vue de sa propre préservation. L'organisme ne se contente pas de se tenir en face de la matière, il ne cesse de la soumettre en fonction de ses diverses activités et autres buts. C'est ainsi, en dernier lieu, que l'organisme atteint une forme d'autonomie et se démarque de son environnement, tout autant qu'il en dépend en raison de la nécessité du maintien de l'homéostasie.

Dans ce contexte, le système nerveux central médiatise l'interface sensori-motrice de l'organisme avec son environnement. Le cerveau ne précède pas la relation entre l'organisme et l'environnement. Au contraire, il ne se développe qu'à la faveur du cadre préexistant d'une telle relation. Les organes périphériques et les surfaces externes de l'organisme servent le but d'échanges continuels et de communication avec l'environnement. C'est dans le cadre de cette interaction à trois termes (système organique, cerveau, environnement) qu'émergent un Soi corporel et une intentionnalité primaire : 
La subjectivité, qui émerge dans le monde avec la formation d'organismes animaux, ne signifie nullement une vie intérieure transmondaine. Au contraire, elle est toujours incarnée et liée à l'environnement, étant présente et agissante en lui. La subjectivité constitue l'aspect intégral des processus biologiques d'un organisme exhibant une auto-organisation et une auto-référentialité, tout autant qu'une relation productrice de sens avec le monde environnant. [...] Ceci se produit à travers les dynamiques du métabolisme d'ingestion et d'excrétion, à travers le cycle de la réceptivité et de l'activité, et pour les primates, finalement, aussi à travers l'interaction d'impressions et d'expressions dans la communication inter-corporelle, faciale et gestuelle (p. 93).

Il en découle que le rapport entre l'organisme et l'environnement ne peut plus être pensé en termes de causalité linéaire dans laquelle un stimulus externe A provoquerait une réponse $\mathrm{B}$ de la part de l'organisme. Fuchs remplace cette forme erronée de causalité par la " causalité circulaire », ou « causalité intégrale », autre moment décisif de son ouvrage. Cette causalité présente à son tour deux formes. La première est la «causalité verticale", et s'articule à l'intérieur de l'organisme, tandis que la seconde prend place entre l'organisme et son environnement. Plus exactement, la causalité verticale est effective au sein des divers niveaux d'organisation interne de l'organisme, de la relation réciproque entre le tout de l'organisme et ses parties les plus microscopiques. Empruntant à Thompson l'expression de « co-émergence dynamique », Fuchs explique comment l'organisme et ses composants (les organes, les cellules, etc.) ne cessent de se co-produire et de se modifier l'un l'autre. Le cerveau agit comme un transformateur au sens où il transforme les états de niveaux supérieurs (comme la sensation, et tout acte intentionnel de la conscience) et les états inférieurs neurochimiques de l'organisme. Ces niveaux ne sont pas isolés les uns des autres, mais ont une influence entre eux. La circularité horizontale, quant à elle, se joue d'abord au niveau du métabolisme et de l'homéostasie, mais également dans une strate supérieure, à savoir au niveau de la relation perceptive et motrice entre l'organisme et son environnement. Le cerveau est encore un transformateur qui agit désormais entre les stimuli et les actions motrices qui sont reliés entre eux dans des schémas neuronaux qui s'activent et s'actualisent en fonction de la perception que l'organisme a de son environnement.

Cependant, la fonction de médiation et de transformation assignée au cerveau serait inopérante sans l'extrême plasticité qui le constitue. En vertu de sa plasticité et de son enchâssement au sein de l'organisme, le cerveau a la capacité d'incorporer l'histoire vécue depuis les niveaux de la vie intra-utérine. Il se développe épigénétiquement en un organe structuré de manière complémentaire avec la vie et l'environnement de l'individu. Ainsi, l'ensemble de nos 
expériences modifie continuellement nos structures neuronales. Cette incorporation de l'expérience dans les structures de la mémoire est fondée dans l'activité fonctionnelle du cerveau qui modifie sans cesse ses propres microstructures. Cette plasticité neuronale assure une transparence des cycles fonctionnels qui ont lieu entre l'organisme et l'environnement. Par ailleurs, cette même plasticité permet une "immédiateté médiatisée ». En effet, la relation entre l'organisme et son environnement est immédiate, elle ne se fait pas au moyen de représentations. Cependant, cette immédiateté se veut toujours médiatisée par les structures cérébrales. Le cerveau constitue un « organe de résonance » qui médiatise l'interface sensori-moteur de l'organisme avec l'environnement.

De surcroît, le cerveau humain est un organe formé culturellement, qu'une personne possède mais qu'elle n'est pas. Cette formation a lieu dès le stade prénatal dans la relation dyadique qui se noue entre la mère et son enfant. Le développement neurobiologique du nourrisson dépend ainsi de manière cruciale de son environnement socio-culturel. Au niveau de l'intersubjectivité primaire, le fœtus baigne d'emblée dans l'environnement de sa mère. Par exemple, il est capable de ressentir à un niveau émotionnel le stress de celleci, ce qui entraîne des conséquences sur le développement de son cerveau ainsi que, ultérieurement, sur sa propre personnalité (p. 177). L'originalité de la démonstration de Fuchs consiste à montrer, expériences à l'appui, comment est corrélée la relation phénoménologique intersubjective qui se noue entre la mère et son bébé avec le développement neurobiologique de ce dernier. Par exemple, une séparation précoce et brutale avec la mère s'accompagne souvent de déficiences ultérieures du comportement et de perturbations d'ordre physiologique. Au stade de l'intersubjectivité secondaire, où la relation dyadique mère-enfant s'ouvre progressivement à une relation triadique incluant désormais une relation à l'objet, apparaît progressivement le langage verbal, lequel ne se défait jamais totalement des gestes corporels que la mère et l'enfant n'ont cessés d'entretenir pour communiquer. Plus généralement, l'apprentissage ne se fait pas sans l'apparition de modèles neuronaux qui sont formés dans la relation à autrui, et qui sont intégrés et réactivés en fonction de la situation. En bref, le cerveau devient l'organe de l'esprit, mais l'esprit lui-même n'est pas dans le cerveau. Les produits culturels ne sont pas des produits du cerveau. Le cerveau s'avère à l'inverse le résultat de tels phénomènes (p. 207).

L'ouvrage se conclut par l'examen des implications de l'approche écologique du cerveau pour la médecine psychologique. Le réductionnisme neurobiologique qui a envahi la psychiatrie a pour fondement un naturalisme exacerbé, à tel point que le trouble mental n'est envisagé que comme un désordre cérébral. Cependant, les processus psychiques ne sont pas réductibles à 
de telles activités cérébrales. A contrario, ils sont toujours incarnés, intentionnels, et plongés dans un champ environnemental de significations. Ils s'enracinent à un niveau intersubjectif et corporel au sein d'un monde de significations partagées socio-culturellement. Fuchs applique soigneusement les concepts fondamentaux de son approche écologique de la cognition incarnée et énactive.

À ce titre, du point de vue la causalité circulaire, tout traitement psychothérapeutique, entendu comme processus interactif et intentionnel, inclut un processus neuronal où les structures cérébrales sont modifiées par l'expérience que le patient a de lui-même et du monde. En retour, la modification neuronale transforme les interactions entre l'individu et son monde. Trois niveaux hiérarchiques ne cessent de s'influencer mutuellement et de se reconfigurer au contact l'un de l'autre. Au niveau macro, nous retrouvons les processus psychosociaux et les interactions entre individus. À un niveau intermédiaire, nous avons affaire aux interactions entre le cerveau, l'organisme et l'environnement. À un niveau micro, enfin, nous rencontrons les processus neuronaux et moléculaires internes au cerveau. Ces trois niveaux, rapportés à l'unité de la personne, incluent donc les causalités verticale et horizontale (p. 255).

Il faut néanmoins reconnaître que le trouble mental relève d'abord et toujours d'une modification de l'expérience vécue du patient. Toutefois, suivant la causalité verticale, cette modification de l'expérience du Soi s'accompagne aussi de perturbations neurophysiologiques. Un patient focalisé sur son état de stress peut bien souvent le faire augmenter et, avec lui, une augmentation du rythme cardiaque, de la chaleur corporelle, etc. Du point de vue de la causalité horizontale, on ne peut pas considérer le trouble mental uniquement comme un dysfonctionnement individuel. La maladie se joue également au niveau de l'intersubjectivité, au niveau macro donc. Fuchs parle de « dysfonctionnements horizontaux», et caractérise le trouble mental comme «un trouble de la communication » (p. 260), ce qu'Erwin Straus, axant sa phénoménologie sur la dimension communicative de l'existence, avait déjà entrepris de faire.

Certes le trouble s'accompagne de modifications biologiques au niveau micro. Toutefois, cette modification n'est jamais la seule cause du trouble, mais bien une conséquence qui accompagne l'expérience subjective et interpersonnelle du trouble. Partant, si l'on considère les troubles mentaux sous l'angle de la double aspectivité de la personne, alors toute action thérapeutique doit être pratiquée suivant les deux aspects, à la fois psychologique et physiologique, et elle relève d'un travail sur les troubles conçus comme des perturbations de l'existence incarnée qui inclut à sa base une biologie 
écologique de l'être vivant. Par conséquent, et nous laisserons le mot de la fin à l'auteur de ce remarquable ouvrage :

La psychiatrie est une médecine relationnelle en un sens englobant: une science et une pratique des relations biologiques, psychologiques et sociales ainsi que de leurs troubles. [...] Ainsi, la psychothérapie ne deviendra jamais une branche de la neurobiologie appliquée. Ses sciences fondamentales essentielles demeurent la psychologie, l'herméneutique, ainsi que les sciences humaines et sociales (p. 276-277).

Remy Rizzo

(Université de Liège)

David Abram, The Spell of the Sensuous. Perception and Language in a MoreThan-Human World, New York, Vintage Books, 1997. Trad. fr. D. Demorcy \& I. Stengers : Comment la terre s'est tue. Pour une écologie des sens, Paris, La Découverte/ Les Empêcheurs de penser en rond, 2013. ISBN : 9782359250626. Prix : 24,50€.

Cette recension demande une première justification : pourquoi parler d'un ouvrage sur l'écologie, dont la version originale date de plus de vingt ans, dans une revue de phénoménologie ? On nous accordera que le sujet écologique est brûlant, que la première traduction française (par I. Stengers et D. Demorcy) est d'ailleurs assez récente : La Découverte décide par ce geste de remettre ce livre d'actualité. Les quelques recensions françaises ont été globalement positives, mais se sont également gardées de commenter l'utilisation de la phénoménologie (Husserl, Heidegger, Merleau-Ponty) dans le cadre de ce questionnement écologique, ce que nous nous proposerons de faire ici.

En anglais et en français, les titres indiquent pourtant une des ambitions traversant le livre : combler un vide trop présent dans le champ écologique, celui de l'esthétique et de la phénoménologie. De nombreux discours sur les catastrophes, présentes ou à venir, nous proposent des conditionnels avec des conséquences chiffrées, mais une telle information reste abstraite et le problème écologique peu senti par certains. À vrai dire, cela constitue déjà, si l'on suit Abram, un symptôme du rapport de nos modes de vie à la nature, rapport fondamentalement non écologique en ce que l'on aurait perdu le lien sensible qui nous unissait au monde plus qu'humain. Le «on» qui perd ce lien, il faudra assumer, avec l'ouvrage, qu'il s'agit de la culture occidentale 
moderne ("l'Occident moderne », «la moderne humanité "civilisée" ", la «culture occidentale » p. 51-52). On sent l'auteur très inspiré par la problématique de Husserl dans la Krisis (trad. G. Granel, Paris, Gallimard, p. 148); on a créé un monde objectif quantifiable, analysable par les sciences, mais on tend désormais à oublier de le ramener à sa source fondamentale : le monde de la vie. Il ne faut pas attendre d'Abram qu'il se tienne fidèlement à la lettre du texte, puisqu'il ne s'agit pas pour lui, à première vue, de redonner un sens existentiel aux sciences par la défense d'une philosophie universelle dont l'état inquiétait le phénoménologue allemand (La crise des sciences européennes..., p. 16-22). Si le décompte scientifique des victimes plus qu'humaines n'a qu'une efficacité limitée, c'est qu'il hérite méthodologiquement d'un oubli auquel il s'agit de remédier en retrouvant ce rapport sensible au plus qu'humain que l'on aurait perdu. On pourra alors à nouveau ancrer nos savoirs et nos modes d'existence dans un monde de la vie entendu en un sens tout particulier par Abram, nous le verrons. L'idée fondamentale est donc que c'est seulement « à l'échelle de nos interactions directes, sensorielles, avec ce qui nous entoure » (p. 341) que l'on pourra à nouveau discerner et répondre aux problèmes écologiques.

La première mention de Husserl dans le texte sert à expliquer l'anesthésie à l'égard du plus qu'humain. En effet, reprenant les analyses de la Krisis sur la mathématisation du monde opérée par Galilée, Abram pose la thèse d'une objectivation excessive du monde opérée par les sciences, en dépassant cependant radicalement Husserl :

Les sciences occidentales et les technologies qui les accompagnent ont oublié le monde de la vie et sa richesse qualitative dont pourtant elles-mêmes dépendent quant à leur signification et à leur existence. Elles ont commencé à envahir aveuglément le monde de l'expérience - et même, dans leur errance, à menacer le monde de la vie de total anéantissement (p. 66).

Cette mathématisation du monde est réinscrite par l'ouvrage dans un mouvement général de notre culture occidentale. Ce mouvement, initié à la fois par les traditions religieuses juives et chrétiennes et par la philosophie à la suite de Platon, aurait systématiquement dévalorisé, au profit des Idées et de l'Esprit, le sensible et le corporel en les traitant comme des ressources passives pour l'Esprit humain, seul véritablement actif face à la nature (p. 73-75, 128-129). Abram termine son analyse du repli sur soi de l'homme occidental par la langue (arbitraire du signe et langue conventionnelle) et l'écriture (développement de l'alphabet). Sciences, dualismes philosophiques, langue et écriture : tout aurait donc été fait, dans la civilisation occidentale, pour construire 
l'homme comme un esprit isolé dans ses techniques et sa technologie, à l'écart du plus qu'humain. Dès Platon se seraient développés les « germes de ce que sera l'éclipse finale de la nature derrière un monde de lettres, de nombres et de textes » (p. 166).

Ces critiques, assez vastes et massives, amèneront sans doute le lecteur à se demander si Abram ne force pas le trait en désignant avec une telle facilité des coupables. Le commentaire de Platon comme d'une philosophie supposant fondamentalement l'écrit (malgré les critiques de Phèdre 274a-277d que l'auteur ne manque pas de mentionner) semble ainsi répondre au besoin qu'a l'auteur de créer une esquisse historique large de nos erreurs. Au fond, le livre, The Spell of the Sensuous dans son titre original, opère un véritable tour de passe-passe. Abram nous installe dans les conditions pour nous faire percevoir une coupure entre humain et plus qu'humain, avant de la dépasser là-même où elle semblait dès lors infranchissable. Car précisément, langue, parole et écriture seront un enjeu fondamental pour retrouver notre rapport sensible au plus qu'humain.

Le tour de passe-passe consiste à justifier la thèse de l'existence d'un fossé actuel entre l'humain et la nature (creusé par nos technologies) sur l'unique base d'un historique de nos constructions technico-conceptuelles occidentales. Bien sûr, on a opposé conceptuellement la nature et l'humain, la culture : cela a donné lieu à certaines pratiques d'exploitation au quotidien. Mais cela suffit-il pour affirmer qu'une rupture d'une telle univocité existe encore aujourd'hui, en dehors de ces concepts par ailleurs largement critiqués ? Cette opposition conceptuelle a-t-elle encore une pertinence réelle, empirique pour tous ces « occidentaux » que nous sommes ? On pourrait se demander si les situations vécues ne sont pas nécessairement plus troubles et complexes : est-il encore possible pour chacun de retrouver une nature plus qu'humaine, intensément sensible, par-delà des techniques humaines, à une époque où il semble devenir impossible de trouver une nature hors de tout lien avec nos techniques (OGM, pollution nucléaire, etc.) ? La question est complexe et terminologique : tant qu'il s'agit de retrouver du plus qu'humain, on peut dire qu'Abram souhaite simplement ne pas voir nos vécus devenir humains, purement humains, sans pour autant défendre la possibilité de trouver en pratique et en théorie un élément purement naturel (il reste un risque de pollution par l'humain en toute chose, mais il y a plus que l'humain). Par moments, Abram qualifie pourtant la nature de "non-humaine » (p. 29, 38, 52), nous enjoignant alors de jouer une dramaturgie de la rupture et des retrouvailles, qui reste étrangement proche du geste de la tradition philosophique qu'il dénonçait. 
Il s'agit de retrouver une « pierre de touche solide » (p. 16), une relation vraie avec le reste de la nature (p. 366). Malgré les critiques virulentes de nos techniques, cela n'implique pas de supprimer le monde que l'humain s'est construit, mais de retrouver le rapport sensible fondamental sur lequel il se fonde. L'ouvrage reste à questionner pour la hiérarchie sensible qu'il opère entre l'humain et le plus qu'humain, notamment quand il compare la richesse sensible de villages tibétains avec la pauvreté des villes états-uniennes : « Ce n'est plus un milieu sensuel (...) mais simplement une absence » (p. 50). Le problème serait celui d'un manque de présence, d'altérité : on perçoit moins d'êtres autres que des humains uniformisés. Et pourtant, cela pourrait être un problème de perception à imputer à l'auteur. Le présupposé central, à questionner ici, c'est que les techniques et technologies diminuent l'échange sensible avec l'autre, qu'elles tendent à mettre à distance ou invisibiliser, comme un voile qui viendrait masquer la nature, à l'instar des «panoramas générés électroniquement » et des écrans qui nous sépareraient du contact incarné avec la nature (p. 16, 52, 90). Or, pour ne prendre que le cas de l'écran comme technique, cette abstraction vis-à-vis du monde de la vie (aussi corporel pour Abram) n'est pas si évidente. À l'instar de Vivian Sobchack, toute une part de l'esthétique phénoménologique mobilise Merleau-Ponty afin de montrer que, par le dispositif de l'écran, le voyant et le visible se reconfigurent lors même de l'acte de perception. Cet acte de perception est en réalité une participation lors de laquelle tous deux ont une part active et qui amène le spectateur à s'impliquer dans une reconfiguration dynamique de son champ d'expérience (A. C. Dalmasso, « Voir selon l'écran (...) », dans M. Carbone, L'empreinte..., Metis Presses, 2013, p. 126-127).

Les critiques parfois hâtives à l'égard de la technique ne doivent pourtant pas masquer que l'auteur a lu Merleau-Ponty et s'en inspire. L'enjeu fondamental du livre est de rappeler que la perception est une interaction, un double ajustement du sensible (lui-même sentant) et du corps sentant (luimême sensible). Dans sa forme fondamentale, l'activité de perception nous enjoint donc d'échanger avec des landscapes, avec le plus qu'humain et son activité propre. Cette interdépendance nous modifie en retour. Au point que le plus qu'humain et le landscape ont eux-mêmes le statut de sujets, vivant leurs propres expériences, nous impliquant dans un dialogue jusqu'à influencer notre langue même (p. 73-86, 93-99, 112-116). L'ouvrage revendique son animisme : le plus qu'humain sent, communique avec nous, agit jusque dans nos pratiques langagières et culturelles. Abram nous montre cette communication dans différentes pratiques étudiées par l'anthropologie (les chants dans le Walkabout ou les histoires chez les Koyukon). 
À cet égard, le changement opéré par l'édition française entre le titre original (The spell of the sensuous) et sa traduction (Comment la terre s'est tue) est pertinent, tout en déplaçant la focale d'une dimension de l'ouvrage insistant sur la magie à une autre, insistant sur l'animisme, thème par ailleurs très important dans les écrits contemporains traitant d'écologie. Un autre écart de traduction - plus discutable - se joue pour des raisons similaires quant au terme de landscape, que l'on traduit généralement par paysage. Dans leur préface (p. 16), D. Demorcy et I. Stengers expliquent le rendre par « milieu » dans un premier temps (p. 28, 37, par exemple) pour éviter de suggérer que le landscape serait inerte, passif, devant notre romantisme contemplatif, avant que leur traduction ne se change en "paysage » dès que Merleau-Ponty apparaît comme une référence principale. Cela pose un problème : le terme français "milieu» recouvre alors à la fois l'anglais landscape et medium (p. 50 ; p. 26 de l'édition originale en anglais), il y a donc ici le risque de perdre une nuance de la langue originale. Il aurait peut-être été intéressant de garder l'ambiguïté française de "paysage » pour traduire l'ambiguïté de landscape dans l'usage d'Abram, qui présente lui-même une attitude d'esthétisation romantique de la nature à laquelle est mêlée une conception plus récente d'un paysage sensible et sentant via Merleau-Ponty.

Le plus qu'humain communique et interagit avec nous. La langue n'est donc pas qu'une abstraction nous éloignant du monde, Abram affirme au contraire que ce monde de la vie qu'il conçoit comme fondamentalement sensuel fournit à la langue sa structure. Se réclamant de Merleau-Ponty quand il refuse de séparer le langage de la parole, il affirme que chaque parole vient modifier le tissu corporel du langage. On est donc pris dans ce tissu comme dans un milieu qui évolue avec notre parole. Abram ajoute alors que, ces mêmes paroles étant enracinées dans les échanges perceptifs et sensibles au sein de milieux plus qu'humains, la langue est dès lors influencée par ces milieux plus qu'humains et est une "expression de la terre animée qui nous enveloppe » (p. 122-123). Dans sa sonorité et sa matérialité sensible, la langue nous permettrait donc de retrouver un contact perdu. Abram différencie deux usages et conceptions de la langue, l'un renfermant l'humain sur lui-même dans un monde sensible appauvri, l'autre le rendant attentif au plus qu'humain. Cette recherche d'un langage originaire, plus proche de la nature, avant sa perversion par notre culture, a de quoi inquiéter. Elle crée un réel danger que n'évite pas l'ouvrage, celui d'opposer rapidement des cultures orales supposées plus proches de l'origine (« ceux qui ont, à l'origine, acquis ce don, la capacité de parler » (p. 335)) à des cultures de l'écrit. 
La phénoménologie tient à vrai dire une place ambiguë dans l'ouvrage. De nombreux thèmes sont abordés, jusqu'aux ekstases temporelles de Heidegger. Elle est donc massivement présente, mais semble parfois être l'objet d'un emprunt biaisé, comme c'est le cas pour la phénoménologie de Husserl (p. 55 suiv.). La problématique du monde de la vie dans la Krisis semble d'emblée tirée vers la philosophie de Merleau-Ponty. Husserl est là pour préparer le terrain, comme on le sent à certaines citations du phénoménologue français, insérées pour affirmer avec insistance que ce monde de la vie est celui d'êtres charnels. Mais surtout, l'auteur nous plonge dans la confusion quand il explique les premiers projets de Husserl, notamment la distinction que celui-ci entendait faire entre la phénoménologie et la psychologie empirique. Abram explique ce qu'est un phénomène à partir d'une définition d'un dictionnaire général (le Merriam-Webster's Collegiate Dictionnary) qui en fait « un objet ou aspect connu par les sens ", la tâche de la phénoménologie de Husserl devenant alors selon lui de « décrire (...) la manière dont les choses se donnent dans notre expérience sensible directe » (p. 59). La confusion est malheureuse, puisque l'on ne sort pas de l'attitude naturelle: et pour cause, le projet d'Abram est profondément différent de celui des Ideen ou des Méditations Cartésiennes. Abram s'intéresse à la perception sensible entrelacée au monde naturel. Il n'opère pas de réduction eidétique ou phénoménologique, et sa phénoménologie n'est dès lors pas la phénoménologie transcendantale de Husserl, qui entendait précisément se distinguer de la psychologie empirique par ces moyens. Le passage par une référence au projet initial de Husserl n'était donc pas obligé pour l'auteur s'il entendait développer une phénoménologie, à moins de créer un malentendu philosophique global. C'est que son approche n'y est pas facilement conciliable. Pourtant, avoir remarqué la nécessité de relier l'écologie à une réflexion esthétique et phénoménologique reste un des mérites de l'ouvrage et un projet d'une importance capitale aujourd'hui. À ce titre, une des questions transcendantales à traiter pour une écologie pourrait être: à quelle condition peut-on avoir conscience d'un changement dans son vécu ? Abram se soucie surtout du contact avec cette pierre de touche sensible qu'est la nature plus qu'humaine, bref d'un changement empirique de nos vécus, qu'il est important de (co-)créer et qui créerait ses propres effets dans les nouvelles relations qui se développeraient. Il s'agit là d'un souci et d'une réponse pragmatiques, pertinents. Cette réponse n'est pas de l'ordre de la phénoménologie transcendantale, qui pourrait poser le problème à un autre niveau, celui de la conscience transcendantale : étant mise entre parenthèses leur dimension empirique, comment avoir conscience de changements dans nos vécus? Que les questions soient posées au niveau empi- 
rique ou par la phénoménologie transcendantale, elles sont d'une égale urgence, et le livre poussera les lecteurs attentifs à penser les deux pour euxmêmes.

Jérôme Flas

(Université de Liège)

Roberta De Monticelli, Il dono dei vincoli. Per leggere Husserl, Milan, Garzanti, 2018, 259 pages. ISBN : 9788811676461 . Prix : $15 €$.

On considère souvent Edmund Husserl comme un auteur hors de portée, non seulement des «non professionnels», mais aussi des philosophes qui n'auraient pas étudié le courant phénoménologique ${ }^{1}$. Il dono dei vincoli (Le don des contraintes), le dernier ouvrage de Roberta De Monticelli, démontre que cette idée n'est pas fondée : la phénoménologie n'est pas une discipline qui, en raison de son type de questionnement spécifique, s'éloignerait de la réalité. Au contraire, selon l'auteure, elle peut être définie comme « ontologie du concret » et de tous les aspects qui concernent l'homme ainsi que sa liberté. $\mathrm{Au}$ moyen d'une analyse fine et efficace, l'auteure accompagne le lecteur, l'exhorte à devenir lui-même phénoménologue, et lui explique comment Husserl ne s'est pas limité à une simple analyse transcendantale. En effet, en combinant et en dépassant la philosophie des mathématiques, la logique, la psychologie de la Gestalt, et même l'esthétique, il a su mettre en avant l'importance de la confiance qu'il nous faut accorder à notre expérience.

L'un des principaux résultats de la méthode phénoménologique est sans doute celui qui révèle le lien intentionnel reliant le sujet et le monde. Aussi, avec la notion d'intentionnalité, Husserl parvient à décrire l'expérience du monde dans sa pleine concrétisation. Cette affirmation peut à première vue sembler paradoxale, car c'est précisément l'acte qui « met entre parenthèses » les éléments entrant dans le champ de la «factité » qui dévoile la véritable essence des choses. C'est pour cette raison qu'il est légitime de soutenir que le sujet husserlien revêt un rôle constitutif vis-à-vis du monde et de tout ce à quoi il est lié. Cette thèse n'a pas pour but de déboucher sur des arguments idéalistes, qui confineraient le sujet à une position solipsiste et transcendante

${ }^{1}$ Une première version de ce texte, en italien, a été publiée dans La Società degli Individui 64, 2019/1, p. 169-173. Remaniement et traduction française pour cette seconde version par Remy Rizzo. 
par rapport au réel, ni même réalistes, mais elle tend à aller au-delà de ces deux orientations en faveur d'une corrélation entre le monde et une conscience qui, comme telle, est unique et concrète ; en d'autres termes : une conscience temporelle, intersubjective et incarnée.

Contrairement à Kant, Husserl affirme la nécessité de passer des objets aux données, car il estime qu'il n'est pas possible d'analyser les fonctions du sujet indépendamment de l'objet. Pour Husserl, la conscience elle-même est une stratification des opérations actives et passives qui constituent l'objet. De la sorte se configure une nouvelle vision du sujet qui, au même instant, se trouve dans la situation paradoxale d'être à la fois sujet et objet de connaissance. L'expérience devient intuition et vision eidétique, elle met le sujet en relation réelle avec le monde, elle anime sa conscience. L'importance de l'intuition, comme le note l'auteure, apparaît déjà dans l'Introduction à la première édition des Recherches logiques, dans laquelle Husserl soutient que les concepts eux-mêmes doivent être issus de l'intuition, à partir de certaines expériences.

Le phénoménologue montre comment nous pouvons, par la logique, régresser vers la sphère des noyaux individuels en relation avec un univers réel. À travers ce processus régressif, qui conduit à un niveau antéprédicatif, il en résulte que la logique postule une théorie de l'expérience, pour laquelle la logique est nécessaire afin de maintenir la formalité : entre l'expérience et la logique existe un rapport de co-dépendance. Le terrain transcendantal se configure comme la base d'une ontologie formelle (au moyen de la logique), nécessaire à la totalité de la réalité. La subjectivité transcendantale est, en ce sens, le lieu originaire de la formation de l'ensemble des sciences, le terrain sur lequel nous justifions la genèse du caractère a priori du monde de la vie. L'idéalité et l'expérience peuvent donc être définies comme deux pôles qui réfèrent l'un à l'autre. Le sujet est en effet irréductible à une seule de ces deux dimensions. Le sujet husserlien se caractérise par une sorte de dualité. Il est à la fois objets parmi les autres objets, un Körper soumis aux lois de la causalité naturelle, mais il assume de surcroît une nature transcendantale, car il est capable des plus hautes fonctions cognitives et de répondre à des lois motivationnelles. Dès lors, il n'est ni possible d'absolutiser ses facultés (comme le suggère l'idéalisme), ni de considérer la réalité comme entièrement indépendante (ce qu'assume la thèse réaliste). La dichotomie classique entre idéalisme et réalisme est simpliste et réductrice : les deux approches ne peuvent pas rendre compte de la complexité du sujet. Le sujet et le monde ne constituent pas deux réalités autosuffisantes et étrangères l'une par rapport à l'autre. Il existe bien plutôt une corrélation profonde entre les deux, une relation dynamique qui les rend mutuellement nécessaires. 
Par conséquent, la conception de l'individu par la philosophie husserlienne dépasse à la fois la dichotomie sujet-objet ainsi que leur simple corrélation : les processus d'incarnation (et donc de mondanéité) et de possession du monde (Welthabe) sont originairement simultanés et interconnectés. Plus précisément, et quant à la possibilité pour le sujet d'être conscient de luimême, si l'on met l'accent sur le rôle de l'intentionnalité (où la conscience est toujours conscience de quelque chose d'autre qu'elle-même), on risque d'aboutir à une interprétation dichotomique qui comprendrait essentiellement le pôle de celui qui comprend et le pôle de ce qui est compris. Cependant, Husserl parle de l'existence d'une conscience de soi pré-réflexive, c'est-à-dire passive, anonyme, et précédant toute activité synthétique. De ce point de vue, on constate la dette de Husserl envers la philosophie antique. L'auteure souligne à plusieurs reprises cette consonance avec la pensée socratique (surtout en ce qui concerne le rôle du philosophe dans la société). Mais nous pouvons aller plus loin encore et soutenir que Husserl partage également certaines thèses d'Aristote qui, dans son Éthique à Nicomaque, déclarait: «Celui qui voit a conscience qu'il voit, celui qui entend, conscience qu'il entend, celui qui marche, qu'il marche, et [...] pareillement pour les autres formes d'activité il y a quelque chose qui a conscience que nous sommes actifs, de sorte que [...] avoir conscience que nous percevons ou pensons est avoir conscience que nous existons (Aristote, Éthique à Nicomaque, IX, 9, 1170a, trad. J. Tricot, Paris, Vrin, 1967, p. 467) ». Le Stagirite parle de l'existence d'une sorte d'auto-référence interne, par laquelle je suis conscient de moimême de manière permanente.

Suivant un raisonnement similaire, dans ses Recherches logiques, Husserl soutient que l'expérience de l'acte intentionnel a lieu avant la réflexion et la perception de l'objet intentionnel. Dès lors, bien que je ne perçoive pas l'acte (il sera thématisé ultérieurement par la réflexion), j'en suis pourtant conscient, grâce à la conscience de soi pré-réflexive : «Une conscience interne, non pas parce qu'elle est une sorte d'introspection, mais parce qu'elle appartient inextricablement à la structure même de l'acte. (D. Zahavi, «SelfAwareness and Affection », in N. Depraz et D. Zahavi (éd.) : Alterity and Facticity, Dordrecht, Kluwer Academic Publishers, 1998, p. 209). Avant que je ne puisse analyser mes expériences, celles-ci sont vécues. Si je perçois l'objet, je suis aussi conscient de ce que je suis en train de faire. Et pour cette raison, nous pouvons affirmer que l'intentionnalité implique nécessairement la conscience de soi.

En cette dernière, l'activité et la passivité s'entremêlent, de même que l'affection et la réflexion. Celle-ci, en effet, suppose une motivation qui, dans le cas de la réflexion sur le Soi, consiste en une auto-affection a priori: « Je 
peux me thématiser, car je suis déjà passivement conscient de moi-même, je peux me saisir, car je suis déjà atteint par moi-même [...] et, bien entendu, cette auto-alimentation de base ne résulte pas d'une activité intentionnelle, elle ne doit pas être activée, contrôlée ou choisie par moi, mais [elle est] un état de pure passivité (D. Zahavi, Self-Awareness and Affection, op. cit., p. 209).

D'un point de vue théorique, si ce qui émerge est l'image d'un sujet imprégné d'activité et de passivité, et nécessairement lié à l'expérience, il est manifeste d'un point de vue méthodologique que Husserl combine l'abstraction de la pensée mathématique avec le caractère concret de l'expérimentation psychologique. Les thèmes traités dans les différents chapitres de $\mathrm{Il}$ dono dei vincoli reflètent ainsi cette diversité. Et de fait, l'auteure s'attarde sur la question ontologique du tout et des parties (qu'elle décrit comme une holologie), sur la corrélation entre la structure sémantique et la contrepartie pratique, et associe raison logique et actualité de la raison pratique. Le fil conducteur de ces domaines, apparemment éloignés, caractérise en réalité ce que l'on entend par « contraintes » : les données, les essences, qui sont à la source de la normativité. La fondation unitaire rend chaque entité capable d'une existence indépendante : l'aprioricité et la nécessité sont inhérentes aux données de l'expérience, et elles structurent l'individu, l'organisent, le rendent concret. La perception s'accompagne du champ d'action, et les lois ne sont pas seulement considérées comme des instruments de logique, mais elles animent aussi la pratique.

En ce sens, De Monticelli soutient que la logique elle-même constitue le cœur de l'éducation humaniste, puisqu'elle nous enseigne le poids (ou la valeur sémantique) des mots, nous éduque à une responsabilité dans l'utilisation du langage, procure aux lois une validité, et rend le doute du philosophe non pas simplement logico-épistémologique, mais bien éthico-juridique. La raison logique et la raison pratique travaillent de concert, se déployant dans la vie intentionnelle du sujet et faisant de la philosophie une question de praxis plutôt que de spéculation transcendantale. L'interprétation de De Monticelli est donc très éloignée des interprétations canoniques de Husserl, et elle parvient à saisir ce que le Maître de la phénoménologie désirait ardemment : la clarté. La phénoménologie — dont la méthode est minutieusement décrite par l'auteure, laquelle invite les « non experts » à adopter et à exercer l'Épochè, la description phénoménologique et la réduction eidétique - nous apprend à voir, à découvrir les propriétés des expériences qui en font des modes de présence des objets, et non de simples états mentaux du sujet conscient. C'est précisément pour cette raison que l'expérience du réel (Erfahrung) diffère de la simple imagination. En effet, en soulignant la contribution que Husserl a apportée au champ de l'esthétique, l'auteure nous confronte à la priorité et au 
caractère unique de l'expérience vécue. La fonction de l'Épochè n'est pas d'ignorer ou d'exclure le réel, mais de suspendre une certaine attitude à son égard, d'abandonner les perspectives prédéterminées, et d'intuitionner l'essence. Dans La Krisis, Husserl soutient que le but ultime de la phénoménologie transcendantale cherche à explorer l'expérience et, par réduction, à analyser de manière systématique la corrélation entre le sujet et le monde. La phénoménologie offre donc les outils pour une réflexion philosophique libre de tout dogmatisme naturaliste. En particulier, la vision eidétique nous permet d'identifier les matériaux a priori et nécessaires à une cartographie des régions ontologiques. Par ailleurs, elle rend le sujet destinataire pleinement capable de prendre l'expérience au sérieux et d'être libre d'acquérir par lui-même une position dans le monde.

Les mots-clés de cet ouvrage pourraient en effet être identifiés à liberté, contraintes, responsabilité. Ce sont précisément les contraintes qui nous rendent libres, c'est la conscience normative intrinsèque à chaque acte qui motive l'action. La raison logique et la raison pratique forment une union inséparable. Mais être libre, c'est aussi être responsable. C'est pourquoi, et surtout dans la dernière partie de l'ouvrage, l'auteure dépeint l'image d'un philosophe qui est éloigné du simple spéculateur intellectuel. À ce titre, selon Husserl - et aussi d'après notre auteure, dont le message d'engagement civil et social devient prégnant - la philosophie n'a pas seulement une origine pratique, mais elle a également pour ambition de construire les racines et les institutions de la société civile. Ces racines se situent dans les discussions et dans l'engagement à la première personne, par lesquels le philosophe advient comme un fonctionnaire de l'humanité, doté du don le plus précieux : le doute, la capacité critique. En fait, la question possède une fonction révolutionnaire, celle d'identifier le sens de l'expérience et de l'action axiologiquement motivante.

De ce point de vue, les textes que De Monticelli met en annexe - la lettre de Bobbio à Enzo Paci, le commentaire de Husserl intitulé Shaw und die Lebenskraft des Abendlandes, et l'essai L'Europe désemparée de Musil sont significatifs et choisis avec pertinence. Ils soulignent la valeur civile de la phénoménologie, laquelle doit devenir une manière habituelle de vivre et, guidée par la juridiction de la raison, une recherche de la vérité. Exposée à l'expérience, elle détermine l'être et rend le sujet responsable de ce qu'il devient : le lien entre les différentes expériences n'est donc pas simplement causal, mais il est déterminé par des contraintes motivationnelles axiologiquement connotées.

Selon nous, le message de De Monticelli est une invitation à voir, à saisir la responsabilité engagée par la liberté que la raison nous accorde. La méthode 
husserlienne concerne donc chacun d'entre nous, et peut être appliquée à différents domaines, passant de l'éthique à la politique, des émotions à l'axiologie. La phénoménologie devient alors une pratique déterminante dans le cadre d'une éducation humaniste et dans la vie de toute personne qui, en tant que telle, prend position dans le monde, le voyant et l'expérimentant d'une manière pleinement authentique.

Valeria Bizzari

(Universität Heidelberg, Fondation Fritz Thyssen)

Giovanni Stanghellini, Matthew Broome, Andrea Raballo, Anthony Vincent Fernandez, Paolo Fusar-Poli, \& René Rosfort (éds.), The Oxford Handbook of Phenomenological Psychopathology, Oxford, Oxford University Press, 2019, 1187 pages. ISBN : 9780198803157 . Prix : $113 €$.

Les premiers mots de cet imposant volume, dont l'objectif est de fournir une synthèse contemporaine d'une discipline émergente annoncée dans son titre, sont révélateurs de l'«atmosphère » qui se dégage de l'ensemble de l'œuvre : la remise en question des évidences. Les éditeurs commencent, en effet, par une (re)définition de la psychopathologie, tâche trop rarement entreprise, et pourtant indispensable pour poser rigoureusement les fondements d'une compréhension théorique et pratique de la clinique psychopathologique.

Cette remise en cause des évidences est notamment celle des savoirs cristallisés : «La psychopathologie phénoménologique n'est pas une des nombreuses approches visant à conceptualiser les troubles mentaux — telles que la psychanalyse ou les sciences cognitives. La psychopathologie phénoménologique développe un cadre de travail pour approcher la maladie mentale dans lequel les hypothèses théoriques sont minimisées, et les formes et contenus de l'expérience subjective du patient, priorisées » (p. 3). Cette perspective en première personne - ou, pourrions-nous écrire, centrée sur la personne - s'accompagne d'une prise de conscience d'elle-même : cette emphase sur la subjectivité, si elle peut ressembler à un engagement théorique, est avant tout le produit d'une praxis cherchant à respecter le phénomène plutôt qu'à s'imposer à lui. Ainsi, les propositions de la psychopathologie phénoménologique, et dès lors celles de l'ouvrage dont nous traitons, se veulent « un langage partagé qui permet aux cliniciens aux différents bagages théoriques de se comprendre lorsqu' ils s'occupent des troubles mentaux » (p. 3). 
Cette ambition d'un langage partagé se concrétise tout de même - on pourrait y voir, à première vue, un paradoxe - par le biais de concepts dans la troisième section. Ce segment procède en fait à une présentation des concepts clés à la faveur d'une tension entre reprise de notions habituellement en usage en psychologie, en psychiatrie et en neurosciences, d'une part, et redéfinition/clarification de ces notions à la lumière du point de vue phénoménologique, d'autre part. Le retour aux choses-mêmes, aux phénomènes, et le primat du réel sur la théorie ne semblent donc pas consister ici en un refus des concepts mais, visiblement, en un processus constant d'information et de remodelage des concepts à la lumière de la rencontre clinique.

Nous comprenons à la lecture de l'ouvrage que la critique permanente (sublimée en l'occurrence par le présupposé que la théorie est toujours-déjà désuète dans la rencontre avec l'altérité du patient) est envisagée comme étant constitutive de l'approche phénoménologique et de son histoire, même lorsque sont abordés les marges et angles morts de ces dernières. On pensera particulièrement au chapitre consacré aux critiques énoncées par Jacques Derrida, Michel Foucault et Gilles Deleuze à l'encontre de la phénoménologie. L'intégration d'une pensée de la clinique en situation, à travers des références antipsychiatriques comme Franco Basaglia, Frantz Fanon ou Ronald D. Laing, sera également à envisager à la lueur de ce leitmotiv. C'est peut-être là l'une des plus grandes originalités de l'ouvrage que d'assumer cet ancrage autoréflexif et concerné par les problématiques d'une anthropologie phénoménologique, caractérisant les travaux de Giovanni Stanghellini, premier éditeur, dès son ouvrage Disembodied Spirits and Deanimated Bodies: The Psychopathology of Common Sense (OUP, 2004, en cours de traduction en français).

Dans un contexte prônant une perspective en première personne associée à une préoccupation écologique, on pourra regretter l'absence de dialogue en tant que tel avec la pensée d'un auteur tel que Carl Rogers ou avec l'approche systémique (et des penseurs comme Gregory Bateson ou Paul Watzlawick, ce dernier s'étant par exemple trouvé en étroit dialogue avec diverses sources philosophiques comme Jean-Paul Sartre). On sera également au regret de constater la présence d'un seul et unique chapitre traitant de psychothérapie (alors qu'il est raisonnable de considérer qu'il s'agit d'une des thématiques incontournables de la phénoménologie clinique de demain).

Si la psychopathologie phénoménologique est définie dans une certaine spécificité, et pourvue donc d'un background conceptuel conséquent, cet exercice ne se produit pas au détriment du nécessaire dialogue avec des conceptions plus classiques et générales de la maladie mentale et de sa prise en charge. Le savoir psychopathologique émerge de la relation entre différents champs du savoir (au premier rang desquels on citera la philosophie ou 
l'anthropologie, mais aussi les sciences cognitives ou la psychanalyse, avec laquelle un débat serré est régulièrement alimenté). Le point de vue phénoménologique, au fond assez original, qui s'esquisse, se situe dans une approche éminemment interdisciplinaire naviguant aux frontières de la philosophie phénoménologique, des neurosciences, des sciences humaines et sociales en général. La septième et dernière section, dédiée à un discours sur les liens entretenus par la phénoménologie avec diverses disciplines ou approches, peut être vue comme l'apogée de ce mouvement omniprésent tout au long de l'ouvrage.

Malgré la volonté de définir la phénoménologie comme une méthode plutôt qu'en rapport à un objet, tentative selon nous réussie, ce manuel à caractère largement international fait la part belle à la schizophrénie et la psychose, dans la lignée des origines de la phénoménologie psychiatrique et de la pensée de ses grands auteurs fondateurs. Si ces thématiques sont assez présentes (particulièrement dans la section six), elles ne sont pas envahissantes puisque l'ouvrage permet largement - il semble même s'agir d'un mot d'ordre - de dépasser les horizons originels de la discipline en nous faisant voyager à travers la plupart des diagnostics (ou plutôt modes d'être-au-monde) traversant le champ de la pratique clinique courante (on citera notamment la personnalité borderline, l'hystérie, la psychopathie, les conduites addictives), y compris ceux traditionnellement envisagés comme relevant du registre névrotique. Les sections cinq et six, consacrées à l'abord concret et phénoménologique d'une pluralité de diagnostics issus des pratiques et nosographies « classiques » (DSM et référentiel psychodynamique, notamment), mettent particulièrement en lumière cette observation, suffisamment rare au sein de la psychopathologie phénoménologique «mainstream » pour être soulignée. La notion d'être-au-monde est peut-être l'une des notions les plus représentatives de la spécificité de l'approche : une approche holistique, antiréductionniste, qui tente de s'intéresser à la complexité de la personne en interaction avec ellemême et son environnement, sans nier l'existence de gestalts fondamentales colorant l'expérience de façon décisive. Ce manuel devient alors un lieu où le diagnostic sémiologique se révèle incarné et " subjectif ».

Alors que la structure du manuel et la cohérence interne de chaque essai permettent une lecture isolée de chacun des chapitres, la richesse et la diversité des apports ne donnent pas l'impression d'un morcellement entre épistémologie et fondements philosophiques et éthiques, d'une part, savoirs cliniques et pratiques situées, d'autre part. Par exemple, la question du naturalisme en psychiatrie - l'éventualité qu'un jour, toute connaissance psychiatrique et psychopathologique soit ramenée à une connaissance biologique, programme assigné, rappelons-le, à la psychanalyse dès Sigmund Freud —, ou celle du 
statut spécifique de la psychiatrie parmi les sciences médicales, sont des problèmes pris au sérieux et dont les implications pratiques sont considérées.

Enfin, à propos de cette dimension « clinique », un dernier point, et non des moindres, a particulièrement retenu notre attention. Les éditeurs reprennent à leur compte le programme de Karl Jaspers - figure centrale du volume -, selon qui le psychopathologue doit s'atteler à comprendre avant d'expliquer. Ainsi, la psychopathologie phénoménologique, sans exclure le point de vue causal - ouverture lui permettant de s'adjoindre le concours des neurosciences, de la psychanalyse et d'autres courants développementaux - , empêche d'escamoter ce qu'elle considère, dans le processus de constitution du socle de connaissance psychopathologique de base, être l'étape déterminante : la description. Puisque ce processus enjoint au praticien de suspendre ses a priori pour s'immerger dans les réalités situationnelles et intersubjectives singulières (donc, de rester le plus proche de ces dernières pour en rendre compte fidèlement avant de les digérer via des filtres interprétatifs), la boucle de la remise en question des évidences est ici bouclée.

Fabian Lo Monte \& Jérôme Englebert

(Université de Liège)

Valeria Bizzari, Sento quindi sono. Fenomenologia e Leib nel dibattito contemporaneo, Milan, Mimesis, 2018, 315 pages. ISBN 9788857549200. Prix : $26 €$.

L'épais volume, au titre ambitieux, proposé par Valeria Bizzari, figure émergente de la psychopathologie phénoménologique contemporaine, impressionne d'abord par sa capacité à proposer une " synthèse ouverte » concernant le débat phénoménologique sur le corps vécu. Les apports classiques des phénoménologies princeps (principalement Husserl et Merleau-Ponty) sont énoncés de façon convaincante. Mais là n'est pas l'originalité voulue par l'auteure qui concentre l'attention du lecteur sur le tournant anglo-saxon contemporain (incarné par des auteurs comme Gallagher, Dreyfus, Noë, Zahavi ou Fuchs) de l'embodied mind ou de l'embodied cognition. On appréciera d'ailleurs de ce point de vue le premier chapitre qui retrace une archéologie de la diffusion de la phénoménologie aux États-Unis qui, volonté assumée (la recherche présentée ici était, à l'origine, un travail d'histoire de la philosophie réalisé dans le cadre de la thèse de doctorat de l'auteure), est présentée avant les phénoménologies husserlienne et merleau-pontienne du corps vécu. 
L'idée centrale de cette phénoménologie contemporaine du Leib est de penser le corps ou, plus précisément, d'intégrer la problématique corporelle sans précisément la réduire à un acte de pensée. Le corps n'est plus un amas pulsionnel, pas plus qu'il ne serait qu'une variable biologique ou encore le lieu secondaire et dépendant du siège de la cognition. Le corps qui intéresse Valeria Bizzari n'est pas celui qui est manipulé ; il n'est, d'un certain point de vue, pas cernable : il est, pourrions-nous dire, un indiscutable. Il est cette entité unique, émotionnellement chargée et mue par l'intentionnalité, qui manipule le monde. Il ne s'agit pas tant, pour reprendre les mots de Merleau-Ponty, du corps que j'ai, mais bien du corps que je suis, siège premier de mon expérience. L'enquête que se propose de mener Valeria Bizzari a pour objectif de savoir s'il est possible « d'être », en son noyau le plus profond, sans « sentir ». On rappellera que sentire en italien se traduira tant par éprouver, sentir et ressentir que par entendre, écouter, éventuellement par tâter et goûter. Le but de ce travail est bien d'offrir une réflexion sur les aspects « incarnés » de la conscience et de comprendre comment notre être corporel non seulement détermine de façon princeps la vie subjective, mais est également la condition même de notre capacité à avoir des perceptions, à entrer en interaction et à agir dans le monde. De façon séduisante, l'auteure procède à trois niveaux d'investigation complémentaires : une trame historique, une dimension théorique et un ancrage pratique - et l'on comprend vite que chaque niveau exerce une influence sur les autres. Toutefois, relevons, tant pour la section historique que pour la section théorique, la quasi absence de la phénoménologie sartrienne. Outre le fait que la problématique corporelle est bien centrale dans celle-ci - et que les apports de l'expérience d'autrui dans le vécu corporel développés dans L'être et le néant, ou ceux de l'expérience émotionnelle développés dans l'Esquisse d'une théorie des émotions (traduit en anglais dès 1948), auraient contribué à densifier cette belle étude du Leib - , on soulignera également le rôle déterminant de Sartre dans la pensée philosophique américaine (et plus généralement anglo-saxonne) contemporaine à l'émergence d'une phénoménologie étatsunienne. Citons les contributions d'Oreste Pucciani, Herbert Spiegelberg ou d'Hazel Barnes (ainsi que d'importantes traductions) ou encore la thèse, publiée en 1961, que Fredric Jameson consacre à Sartre, mais aussi plus récemment l'influence de Sartre dans l'œuvre de Shaun Gallagher.

Selon nous, l'audace du volume tient prioritairement dans sa troisième et dernière partie, lorsque la méthode phénoménologique est «mise en pratique », éprouvée notamment dans la relation clinique : celle de la médecine en général, et plus fondamentalement celle du champ de la psychopathologie en ce qu'elle a à traiter des troubles du soi et de l'intersubjectivité. L'objectif est de comprendre comment l'adoption d'une définition incarnée du sujet 
implique une remise en question du dualisme biologie/corps et esprit/psyché et présente d'importantes répercussions sur l'éthique médicale, particulièrement la relation médecin-patient qui doit être valorisée et repensée, en dehors d'un strict échange d'informations objectives, comme une expérience affective déterminante dans l'expérience tant du patient que du médecin. On appréciera tout particulièrement le détour par les propositions que Drew Leder, philosophe et médecin du Maryland, développe dans The Absent Body (Chicago, Chicago University Press, 1990) concernant l'intentionnalité motrice ou le schéma corporel et suggérant que l'expérience de la maladie est une affection corporelle expérimentée par une personne incarnée et en relation avec le monde. Valeria Bizzari est en syntonie avec cet auteur puisque, comme elle, il cherche à extraire du savoir phénoménologique une contribution à une " éthique incarnée » [etica incarnata] et à la rencontre d'un individu situé.

L'intersubjectivité, qui est toujours une intercorporéité, se révèle également déterminante dans de nombreuses affections psychopathologiques (en particulier la schizophrénie et l'autisme). L'hypothèse phénoménologique principale est que ces troubles sont générés par un trouble de la conscience sensorielle de soi. Cette crise de l'expérience préréflexive et du vécu incarné, mise en évidence par l'approche phénoménologique, est discutée à travers les notions d'hyper-réflexivité, de manque de sens commun ou de perte de l'évidence naturelle. Dans une partie plus expérimentale (et très convaincante), la méthode phénoménologique est alors appliquée à un cas concret, celui de M., une personne Asperger (exempte de troubles secondaires cognitifs ou physiques) et de son expérience de l'intersubjectivité.

Avant de révéler le contenu de son expérience clinique, Valeria Bizzari s'attache à démontrer les limites des explications cognitives « classiques » du symptôme, cardinal dans l'expérience autistique, d'isolement relationnel. La célèbre Theory of Mind, partant du présupposé que l'expérience d'autrui repose sur une interprétation et une mentalisation des états subjectifs de son vis-à-vis, est soigneusement critiquée. À prendre au sérieux cette hypothèse, l'intersubjectivité engagerait une production explicite de savoirs qui médiatiserait notre compréhension de l'autre. Pour autant, est-il possible de conclure à l'absence d'investigation intellectuelle de la part du sujet autiste ? Valeria Bizzari, grâce à la rencontre de M., mettra au contraire en évidence qu'au lieu d'être un déficit, la théorisation des états mentaux d'autrui constitue au fond une stratégie compensatoire mise en place en raison d'un trouble relationnel plus fondamental. À ce titre, notons que l'on retrouve ici la même critique que celle énoncée par Till Grohmann dans Corps et Monde dans l'Autisme et la Schizophrénie: Approches ontologiques en psychopathologie (Springer, 2019). Selon Bizzari, la rencontre clinique fait apparaitre que ce que cette 
lecture cognitive orthodoxe considère comme le problème est en réalité la solution mise en place par le sujet autiste pour contourner son problème relationnel fondamental.

On comprend, avec Gallagher - notamment dans How the body shapes the mind, paru en 2005 aux Oxford University Press - que le déficit en Theory of Mind peut difficilement expliquer l'autisme car cette dernière théorie peine, de façon plus globale, à rendre compte de l'expérience intersubjective nonautistique. L'être social n'est pas un scientifique qui observe le comportement d'autrui pour en tirer des théories sur les états mentaux des autres, afin de les expliquer et de les prédire ; son rapport à autrui n'est pas primairement médiatisé par un savoir explicite. À l'inverse, le sujet incarné, mû par l'intentionnalité motrice, s'engage de façon directe et de façon préréflexive dans l'aventure intersubjective sans passer par la médiation théorique/intellectuelle. Nos interactions sont fondées sur des facteurs environnementaux et contextuels, plutôt que sur des attitudes mentales ou conceptuelles, explicatives ou prédictives, et nos rencontres avec les autres ne sont pas basées sur une théorie implicite. L'on met donc en évidence un relatif retard de l'expérience réflexive par rapport au vécu qui repose sur une intersubjectivité primaire incarnée (l'auteure rappelle que Husserl, Scheler et Merleau-Ponty ont insisté sur la centralité des processus prélogiques et souligné l'importance de la rencontre et de l'engagement corporel de celle-ci).

D'un point de vue psychopathologique, les données théoriques et empiriques recueillies par la philosophe convergent vers une conception du trouble autistique en tant que perturbation fondamentale de la perception sociale ancrée. Plus précisément, les données issues de cette enquête théorico-clinique permettent de soutenir que le principal problème de l'autisme ne consiste pas en un déficit de la théorie de l'esprit, mais est plutôt attribuable à une distorsion de l'affectivité et du sens commun - qui est l'accord relationnel tacite permettant au sujet d'entrer en relation avec l'autre de façon immédiate, précisément sans avoir recours à une logique réflexive, voire algorithmique - qui sont tous deux liés au sens préréflexif et corporel de soi.

Puisqu'il s'agit d'un trouble qui concerne le sens préréflexif et corporel de soi, Valeria Bizzari décide de réaliser une investigation qualitative, qui étudie les structures de la subjectivité des sujets affectés (à travers l'étude des existentiaux que sont l'espace, le temps, le soi, le corps et l'autre). À la méthode thérapeutique de prise en charge de l'autisme DIR (Developmental, Individual-Difference et Relationship-based), développée par Greenspan dans The Growth of the Mind and the endangered origins of intelligence (Reading, Addison Wesley Longman, 1997), Valeria Bizzari ajoute le E d'Embodied. Elle traitera plus en profondeur ce sujet dans un article intitulé From DIR to 
DIRE: the role of embodiment in the treatment of self-disorders (in J.G. Pereira, G. Gonçalves, V. Bizzari (Eds), The Neurobiology-PsychotherapyPharmacology Intervention Triangle: The need for common sense in 21st century mental health, Vernon Press, 2019). La conscience du moi corporel permet au sujet de développer ses facultés motrices et, secondairement, ses facultés cognitives. De façon très concrète, l'auteure explique la mise en place d'exercices de compétences relationnelles (modèle du floor-time par exemple), ainsi que d'exercices visant à renforcer le schéma corporel. C'est alors le travail de la philosophe américaine Maxine Sheets-Johnstone qui, notamment dans The Primacy of Movement, Expanded second edition (Amsterdam, John Benjamins Publishing, 2011), est la source permettant d'assumer le pôle incarné de la prise en charge thérapeutique, grâce à ses propositions sur la danse et le mouvement. Convaincue que la compréhension des pratiques corporelles est fondamentale pour le bien-être du sujet, Sheets-Johnstone suggère une approche se concentrant sur le rôle du mouvement, considéré comme le résultat d'une combinaison de l'espace, du temps et de la force du corps. La pratique d'exercices corporels comme ceux inclus dans la danse est un outil permettant de renforcer la conscience de soi et de ses potentialités, de (re)prendre contact avec son corps et, par conséquent, de renforcer de nombreuses caractéristiques de la subjectivité. Ce projet de «thérapie incarnée » s'avère important pour le développement de l'émotivité, en renforçant le processus de résonance corporelle permettant la compréhension préréflexive de l'altérité et facilitant ainsi l'expression de fonctions qui, dans des pathologies comme l'autisme, sont compromises.

En conclusion, que retenir de cette étude du corps vécu ? En plus d'une contribution originale et conséquente au champ de l'Embodied cognition cet apport parmi d'autres rendant sans doute impossible de continuer à considérer l'humain cognitif comme on le faisait de façon dominante il y a quelques années encore dans le champ de la psychologie et de la psychiatrie - , on soulignera l'audace méthodologique de pratiquer une philosophie elle-même incarnée. La philosophe en situation propose une nouvelle manière de faire de la philosophie, celle d'une construction d'une philosophie appliquée, brouillant ainsi les pistes disciplinaires de façon heuristique : si, comme le suggère John Cutting, la psychopathologie est une "philosophie appliquée » (Principles of Psychopathology: Two Worlds, Two Minds, Two Hemispheres, Oxford, Oxford University Press, 1997), lorsque le philosophe « joue le jeu » d'être en psychopathologie, il propose de nouveaux savoirs et trouve des matériaux inédits permettant de combler l'envie de Bachelard : «Bien souvent, poursuivant dans les livres notre travail solitaire, nous avons envié les psychiatres auxquels la vie offre chaque jour des "cas" nouveaux, des "sujets" 
qui viennent à eux avec un psychisme complet » (La terre et les rêveries $d u$ repos, Paris, Corti, 1948, p. 91). Penser le corps incarné avec son propre corps incarné ; il s'agit là d'une manière de faire de la philosophie qui, s'inscrivant dans les pas de nombreuses contributions incontournables, réinvente une fois de plus son propos.

Jérôme Englebert (Université de Liège)

Patrick Chemla (dir.), Le Collectif à venir. Psychiatrie, psychanalyse et psychothérapie institutionnelle, Erès, Toulouse, 2018, 248 pages. ISBN : 2749258731. Prix : $25 €$.

\section{Pour une compréhension phénoménologique du collectif}

Le Collectif à venir est un recueil publié en réponse à la réforme française des groupements hospitaliers de territoire (GHT) issue de la Loi santé de 2015. Des psychanalystes, psychologues, infirmier.e.s, cadres de santé, docteurs en psychologie, philosophes, psychiatres, chargés de collections d'art, directeurs, internes en psychiatrie, médecin-chef, etc. prennent la parole chacun à leur tour sur le thème du collectif. L'engagement politique de l'ouvrage est explicite sous la plume du directeur du Collectif à venir : "Contre les folies évaluatrices et les volontés de mise au pas de la Haute Autorité de santé, qui s'institue aujourd'hui en "police de la pensée" du soin et des pratiques ", il s'agit « de relancer sans cesse la création de lieux d'accueil et de soins qui s'appuient sur la créativité et la parole mise en acte de ceux qui s'y tiennent : patients, soignants, mais aussi familles et personnes concernées » (p. 8).

Depuis 2016, la réforme des GHT bouleverse le système de santé français en proposant « un regroupement des établissements publics de santé au sein de territoires avec l'objectif affiché de mutualiser les ressources et d'améliorer l'accès à des soins de qualité au bénéfice de la population $»^{1}$. Devant ce remaniement séduisant sur papier, certains restent méfiants et s'interrogent quant à sa traduction sur le terrain. D'autant plus que seuls les acteurs des hôpitaux publics semblent avoir été impliqués dans les discussions autour de la réforme pour rester au plus près des réalités du terrain : aucun citoyen ni représentant associatif n'a vraisemblablement été consulté. Dès le 8 
juillet 2016, Claude Rambaud, alors présidente du CISS (Collectif Interassociatif Sur la Santé), situe la réforme des GHT « dans une vieille conception de l'action publique qui voit l'administration décider pour la population, sans la consulter, de ce qui est bon pour elle $»^{2}$. À cette critique, une autre s'impose dès que sont mises en lumière les situations engendrées par certains regroupements hospitaliers inadéquats pour les usagers. En effet, le rattachement de certains établissements à un territoire plutôt qu'à un autre rend parfois aux usagers l'hôpital plus difficilement accessible, ce qui éloigne paradoxalement les services de soins de santé. La réforme des GHT, loin de faire l'unanimité, constitue une occasion pour les auteurs du Collectif à venir de relancer un débat autour du concept de collectif. En matière de soins de santé, ce concept est porté par la psychothérapie institutionnelle et est volontiers attribué au psychiatre français Jean Oury (1924-2014). La mort récente de ce dernier ainsi que la reconceptualisation de la notion de collectif proposée par Olivier Apprill constituent le point de départ des différentes propositions des auteurs de l'ouvrage.

Cinq parties structurent Le Collectif à venir. La première esquisse l'horizon théorique et politique du propos. Olivier Apprill, psychanalyste, inaugure le recueil en rendant compte du concept de collectif chez Oury (p. 1730). Ce faisant, il pose le cadre de l'ouvrage puisque c'est à partir de sa reconceptualisation de la notion de collectif que les autres auteurs travaillent. À sa suite, Serge Klopp, infirmier cadre de santé, prend position contre la loi santé de 2015 qu'il définit comme « la loi la plus méconnue aux conséquences dramatiques pour la psychiatrie de secteur » (p. 47). Cette loi est, dit-il, « la remise en cause du principe [même] de continuité [des soins] » (p. 49). La deuxième partie de l'ouvrage prolonge cette critique et met en évidence, précisément, l'importance d'une continuité — du soin d'abord, mais aussi d'une présence (à soi) et du lien (p. 67-77). À cette continuité doit se joindre une ouverture à " l'improviste, la surprise, la déprise, l'incongruité, le rire » (p. 82). La difficulté de faire co-exister les deux - continuité et déprise - est illustrée par l'anecdote d'une promenade avec des patients racontée par Blandine Ponet, infirmière. Cette anecdote sera détaillée plus loin pour les interrogations qu'elle soulève.

La question de la déprise, autrement dit de l'improvisation dans le soin, est ressaisie dans la troisième partie en envisageant l'œuvre comme la création d'un espace, l'émergence d' ' un lieu pour l'indétermination comme socle du lien et d'une existence habitée » (p. 143). Nader Aghakhani, psychologue, définit l'atelier (de création au sens large) comme un " espace de rencontre » où «l'artiste, l'analyste et bien d'autres se rejoignent» (p. 125). Parallèle- 
ment, l'atelier est défini comme un «lieu à tenir » dans la mesure où il accueille « un certain nombre d'actions, d'exercices, de schèmes [qui, toujours, sont] à répéter, à reprendre » (p. 125). Dans ce lieu de rencontre qui est à tenir, « il s'agit de maintenir une ambiance pour installer un rythme » et « laisser venir... la construction, l'élaboration, le lien social, la liberté [...]»(p. 125). On sait que l'ambiance est une question phénoménologique importante. La quatrième partie, intitulée «Au fil de l'expérience », rassemble un ensemble de pratiques - entre club thérapeutique, clinique de rue et cas cliniques. Deux éléments cruciaux au moins ressortent de cette partie. Tout d'abord, s'impose l'idée que le club n'est jamais fait une fois pour toutes et qu'il peut, à tout moment, s'atrophier s'il n'est pas animé par une ambiance. Le propos de Jean Oury est rapporté par Madeleine Alapetite, cadre de santé, pour expliciter l'enjeu du club :

Le club n'a de sens que si on tient compte de sa finalité, responsabiliser les gens. Dans une collectivité, il faut toujours alimenter le feu, c'est ce que disait très bien Tosquelles : ça nécessite une ambiance vivante permanente et ça se nourrit avec des sentiments, avec de la parole, avec des gens qui sont là... sinon ça s'atrophie (p. 175).

La quatrième partie de l'ouvrage insiste aussi sur la confiance que doivent susciter les lieux - il est fait mention du club mais il est possible d'envisager les ateliers de création en tant que tels : ces lieux doivent en permanence dégager une atmosphère vivante, une ambiance telle que chacun se sente en sécurité et en confiance. Le message de la cinquième et dernière partie de l'ouvrage est explicite depuis son titre : " "Voyageurs! Il n'y a pas de chemin, le chemin se fait en marchant le chemin se fait en marchant" (extrait tiré des Champs de Castille d'Antonio Machado) ». Le chemin inexistant dont il est question est celui qu'entendent emprunter les auteurs du Collectif à venir. Mais s'il s'agit d'un chemin non tracé, il est toutefois pensé dans la continuité de la démarche de Jean Oury. L'absence de tracé prédéfini est absolument fondamentale dans la perspective des auteurs car, dans les faits, «c'est la rencontre avec ce que l'on ne cherche pas qui permet des avancées souvent cruciales et essentielles » (p. 196). Ainsi l'absence de tout tracé apparaît-elle comme une exigence méthodologique qui fait écho à l'imprévisibilité et, par-là même, à l'inachèvement, absolument nécessaires, qui caractérisent le projet même de la machine du collectif (p. 244).

Afin d'en saisir la portée concrète dans les soins, le collectif tel qu'il est présenté et mis en scène dans le Collectif à venir doit être mis au travail. L'enjeu principal est de prolonger l'idée, suggérée par Olivier Apprill au début 
de l'ouvrage, selon laquelle le collectif — invisible et insaisissable — n'est qu'un «"lieu" qui n'existe qu'au niveau logique » (p. 30). Par un biais phénoménologique, il s'agit de montrer que le collectif peut, s'il est nourri et vivant, se faire sensible et ainsi dépasser le seul niveau logique. Le collectif ne peut, dans ce cas, être réduit à une stricte abstraction. Penser phénoménologiquement le collectif permet de ressaisir successivement, comme on va le voir, des enjeux tels que celui de l'ambiance, des lieux, du projet du collectif — voire des collectifs - et de l'altérité. L'intérêt d'une reprise phénoménologique de ces motifs est, à la fois, de considérer les propositions du Collectif à venir à partir du rapport à soi, aux autres et au monde, et de mesurer effectivement le caractère éminemment politique de l'ouvrage.

\section{La phénoménologie sartrienne comme origine de la notion de collectif}

Initialement, Jean Oury pense le collectif de manière péjorative. Influencé par le Sartre de la Critique de la raison dialectique, il associe le collectif au sériel, à l'idée d'engluement et de passivité. Il fait correspondre le collectif à l'aliénation de la liberté. À cette époque, Oury oppose le collectif au groupe en fusion qui, pour sa part, renvoie à une liberté créatrice. La particularité du groupe en fusion étant de se produire toujours sur la base d'un collectif, il est possible de relier les deux termes et de parler, avec Oliver Apprill, d'une « dialectique circulaire infernale» (p. 24) : le groupe doit faire un effort pour s'arracher du collectif mais il ne peut éviter le risque de retomber dedans. Le groupe est continuellement menacé de retourner d'où il vient, de se dégrader à nouveau dans le collectif. Cette compréhension du collectif est amorcée par Oury en 1960, au cours des séances du GTPSI (Groupe de Travail de Psychothérapie et Sociothérapie Institutionnelle). Ce groupe est alors à l'avant-garde de la réflexion psychiatrique au niveau de la parole collective : entre 1960 et 1966, il réunit les principales figures de la psychothérapie institutionnelle autour de François Tosquelles et de Jean Oury à raison de deux ou trois rencontres par an. Mais chemin faisant, Oury développe une autre compréhension du collectif. Dans un séminaire, en octobre 1994, alors qu'il cherche à faire comprendre son concept, il raconte :

Étant petit, $[\ldots]$ j'étais très influencé par une machine $[\ldots]$. C'était une machine un peu surréaliste, c'était une machine à coudre [...]. Une vraie, avec des pédales, des courroies, $[\ldots]$ avec une petite couille en acier qui bouge $[\ldots]$. Le résultat de tout ça, avec des grandes roues, des pédales, des courroies, le pied qui remue, etc. ? Et bien le miracle s'accomplissait à chaque fois, avec une 
seule aiguille qui piquait point par point. C'est pour ça que je suis à La Borde ! Je vais reconstruire une machine à coudre collective fantastique. [...] (p. 30).

Un changement de direction est ainsi engagé. Oury passe d'une conception négative à une conception résolument positive du concept de collectif. Ce dernier lui apparaît dorénavant « incontournable pour toute praxis psychiatrique institutionnelle» (p. 25). Mais Olivier Apprill rappelle :

On ne peut pas [...] voir un collectif ni l'attraper. Ce n'est pas un groupe réel considéré dans ses dimensions sociales, interrelationnelles, voire inconscientes, on ne peut pas l'objectiver : c'est un « lieu » qui n'existe qu'au niveau logique, une catégorie abstraite, un champ transcendantal pragmatique, c'est-à-dire ce qui constitue la condition de possibilité pour l'émergence d'une situation pratique où le désir, et donc le sens, peuvent circuler. [...] le collectif est aussi un concept d'efficacité politique pour la mise en place d'un milieu, un outil fondamental pour une politique de la singularité, donc une politique de la folie. Produire du collectif, c'est produire des conditions de possibilités (p. 30).

Derrière le collectif ne se cache ni groupe particulier, ni groupe de travail, ni collectivité. En tant que «machine abstraite» (p. 27), le collectif joue strictement un rôle de condition de possibilité dans la pratique des soins. Loin de contester l'idée selon laquelle le collectif se situe au niveau logique, il s'agit plutôt de la relancer pour la prolonger. Pour cela, l'image de la machine à coudre est à prendre au sérieux, car elle permet de comprendre une première fois ce que peut être une «machine abstraite» comme celle constituée par le collectif.

\section{Le collectif comme machine sensible}

La description qu'en donne Oury laisse entendre qu'un mécanisme aux rouages complexes mais harmonieux habite toute machine à coudre. De fait, toute une mécanique interne se met en branle dès qu'une machine à coudre est mise au travail. Des engrenages se coordonnent sans relâche. L'utilisateur ne le voit pas mais chaque pièce se meut pour faire sa part de l'office et concourir à la naissance d'une création unique. Toutes les pièces de la machine à coudre sont là, présentes, mais invisibles. Elles se manifestent dans leur élan, leur mouvement commun - ou négativement : dans un ratage, quand la bobine de fil s'emmêle, fait des nœuds, ou que l'aiguille se casse. C'est dans ce cadre que le collectif est comparable à une machine abstraite : comme la machine à coudre animée par le dynamisme de ses pièces, le collectif se définit par son 
mouvement. En d'autres mots, le collectif ressemble à une machine à coudre parce qu'il est aussi, dans son concept, habité par un élan commun. Il faut voir que cet élan est fragile, à l'image du mouvement de la machine à coudre qui peut s'interrompre à tout moment. En tant qu'il risque toujours de s'essouffler, de ralentir, voire de s'interrompre, le collectif tel qu'il est envisagé par Oury dans les années 1990 encourt les mêmes risques que le groupe en fusion sartrien de 1960. Le collectif s'expose toujours au risque de retomber, c'est-àdire au risque de la sérialisation. C'est précisément pour cette raison que son élan doit être continuellement repris et relancé, activement pensé et vécu, par l'entremise très concrète d'individualités, dans des moments, des lieux, à travers des échanges, ou durant des activités par exemple. L'image de la machine abstraite proposée par Oury, loin d'écarter la pratique concrète des soins, peut sans difficulté être assumée dès lors qu'on comprend, d'une part, que l'abstraction est précisément ce qui laisse place au concret et, d'autre part, que la machine en question se fait sensible dans chacun des lieux et à chaque fois que le collectif tient en créant une certaine ambiance entre soignants, soignés, etc. Cette ambiance, difficilement exprimable, relève pour ainsi dire de l'intuitif. Sa seule condition de possibilité énonçable est la suivante : elle nécessite des personnes ouvertes disposées à accueillir le sujet derrière chaque individu ainsi que l'imprévu sous toutes ses formes. Cette ambiance correspond à un certain climat, un certain environnement. Elle ouvre un monde à chacun, qui plus est un monde commun. Dans une dialectique sans fin, l'ambiance habite le sujet en même temps que le sujet habite l'ambiance. Ainsi, parlant d'ambiance, une implication réciproque semble pouvoir être suggérée entre un sujet et son environnement, entre un «je » et le monde qui l'entoure. L'ambiance aménagée par le collectif est à comprendre comme un lieu de rassemblement. Tout l'enjeu de ce lieu est de rester en vie; il s'agit donc de le supporter pour le perpétuer. En ce sens, le collectif a beau être une machine abstraite, il doit tenir. Or, l'anecdote de Blandine Ponet met précisément en tension cette idée de machine qui tient (p. 91-99).

Le propos de cette infirmière illustre le sentiment d'éparpillement que peuvent ressentir les soignants en sortie avec un groupe de patients. Elle explique: alors qu'à l'hôpital, les individus forment automatiquement un groupe aux yeux du soignant, ce dernier doit admettre que le groupe ne fait pas évidence à l'extérieur des murs de l'hôpital. En effet, chacun tend à partir de son côté pour explorer une partie du monde. Pour le dire dans des termes sartriens : dehors, le groupe n'est plus en fusion, il se délite. Dans ce contexte, la nécessité de « fabriquer concrètement l'espace-temps et l'être ensemble de la promenade » (p. 92) se fait sentir. Un monde commun est à reconquérir. «Faire une promenade » exige un faire au sens fort du terme. Blandine Ponet 
écrit : «Avec les patients, ce sens de "faire" est entièrement chargé : consistant » (p. 92) puisqu'il s'agit de garder ensemble des individus, autrement dit de créer sans répit un espace, un temps et un être ensemble, c'est-à-dire du commun. Cette anecdote montre que le lien établi par et dans l'espace-temps de l'hôpital, loin d'être évident et acquis, est fragile et continuellement à recréer. Parallèlement, cette anecdote soulève la question de savoir ce qu'on fait dans et avec l'éparpillement, s'il est tenable, et si oui, jusqu'où il peut être assumé ?

Les images de la machine à coudre et de la promenade permettent d'intuitionner ce que peut être le collectif pour Oury et la raison pour laquelle il en parle en termes de «machine fantastique ». Le collectif n'a pas de contours nets car, dans les faits, il se ramène à une ambiance. Le collectif n'est rien de précis : ses rouages sont aussi fragiles qu'invisibles et disséminés. À cet égard, que certains invitent à "l'acceptation d'une certaine confusion» (p. 82) est significatif. Tout se passe comme si le collectif, concept abstrait, coïncidait à une ambiance indéterminée voire indéfinissable. Pourtant, paradoxalement, au fur et à mesure que les pages du Collectif à venir se tournent, le collectif, aussi abstrait et conceptuel puisse-t-il être, semble prendre corps et créer un espacetemps tangible. Du moins est-ce l'hypothèse que plusieurs auteurs de l'ouvrage suggèrent. Dès lors, la notion de collectif se morcelle. Considéré d'abord comme un strict concept logique, puis comme un mouvement donnant naissance à une ambiance, le collectif devient un espace-temps sensible. Dans tous les cas, l'idée selon laquelle il faut relancer ensemble le collectif, c'est-àdire le penser et repenser collectivement, est maintenue. Cet élan général relève de la responsabilité de tous les « soigneux » pour reprendre le terme de Nader Aghakhani - ce dernier utilise ce mot pour éviter les termes « soignants » et « soignés » et désigner toute personne prenant soin d'elle-même, des autres et du social (p. 126). L'hypothèse du collectif comme espace-temps sensible est tirée de la suggestion de voir « au travers de l'œuvre, la création d'un lieu, son émergence » (p. 113). À la suite de cette idée, Le Collectif à venir convoque le lieu constitué par l'atelier. L'hypothèse mise à l'épreuve est la suivante : loin de se réduire à une stricte machine abstraite invisible, le collectif peut, par moment, correspondre à un lieu sensible. Plus précisément : le collectif peut coïncider à un ensemble d'espace-temps consistants.

Précédemment, l'espace-temps de la promenade - lorsqu'il est fait, assuré, et n'est pas qu'éparpillement - était évoqué. Dès à présent, un autre espace-temps est à envisager: celui de l'atelier organisé et pensé par la psychothérapie institutionnelle (l'atelier de théâtre, de musique, d'écriture ou de peinture par exemple.). Nader Aghakhani définit l'atelier comme «un 
espace de rencontre », « un lieu à tenir » qui inaugure une « mise en mouvement » des statuts, rôles, fonctions, et « une circulation » (p. 125). L'atelier est une ouverture dans laquelle "je s'invente et se réinvente» (p. 126). Ici, le « je » en question n'est pas seulement celui du patient. Il s'agit du « je » de tout soigneux. Jean Oury avait l'habitude de dire : «Si je n'ai pas été soigné au moins cinq minutes sur la journée, c'est que je n'ai pas été soignant $»^{3}$. Avec celle de Nader Aghakani, cette proposition bouscule l'apparente évidence selon laquelle soignants et soignés s'opposent pour soutenir, au contraire, une indétermination : dans un hôpital, chacun est soigné et soignant selon les moments, les lieux et les interactions. Le propos d'Oury est une autre manière d'affirmer la dimension relationnelle du soin. C'est exactement dans cette veine que l'espace-temps de l'atelier parait, dans la perspective de Nader Aghakani, pouvoir « tenir lieu de corps » (p. 118) dans la réinvention du sujet quel qu'il soit. Toutefois, il faut ajouter que bien qu'il en ait la possibilité, l'atelier n'ouvre pas systématiquement un tel espace-temps constructif. Pour reprendre les deux images précédemment mobilisées, si aucune machine à coudre n'œuvre ou si personne ne cherche à y faire une promenade, l'atelier peut facilement se réduire à un espace-temps quelconque où il n'est question d'aucune invention ni réinvention. S'il veut offrir au sujet un lieu et un temps où se dire et se faire, l'atelier doit continuellement s'interroger et être interrogé sur ce qu'il fait, comment il le fait et avec qui. Cette réflexivité est indispensable pour espérer faire naître dans un atelier l'ambiance du collectif. Il faut remarquer que l'atelier de création n'est pas le seul lieu capable de rendre sensible le concept de collectif. D'autres espace-temps le peuvent aussi : une salle d'accueil, un jardin, un banc, un couloir, une salle à manger, une salle de sport par exemple. Peu importe finalement l'endroit du moment qu'il est le lieu d'une ouverture à soi, à l'autre, au monde, à l'événement, à la déprise, qu'il est continuellement pensé et repensé, jamais stagnant ou objectivé définitivement, bref : qu'il fait place à l'individualité.

Le collectif peut ainsi bel et bien se comprendre différemment que comme une stricte machine abstraite. Dès lors qu'il est investi, le collectif dépasse le concept pour, ponctuellement, prendre corps. Il s'incarne à des moments et crée alors une ambiance. Tout se passe comme si l'existence du collectif tenait dans la fulgurance d'instants. Dans son concept même, le collectif constitue presque une impossibilité : jamais il ne peut s'établir définitivement car, précisément, il cesse de faire collectif dès qu'il cesse de (se) penser, de se mouvoir, pour s'installer et se figer. Pour le collectif, ne pas être en mouvement, ne pas se ressaisir continuellement, revient à dépérir prématurément. 


\section{Le projet du Collectif à venir : une phénoménologie des collectifs}

Si le recueil prend pour point de départ la notion de collectif, son titre marque un double pas de côté en convoquant l'idée d'un Collectif avec majuscule et d'un «à venir ». Comment comprendre cette double distinction? Patrick Chemla reprend la proposition de Jean Oury - selon laquelle le collectif est un concept en faveur d'une invention et improvisation permanente en milieu de soins - pour la préciser en insistant sur le fait que le projet du collectif est par définition un projet inachevé, voire un projet inscrit dans l'inachèvement même :

Le Collectif à venir [est] cette décision à renouveler à chaque franchissement, une façon de souligner l'inachèvement nécessaire du projet: que serait un projet achevé sinon un projet mort (p. 244)?

Le projet infini du collectif rejoint la conception sartrienne de la conscience - la conscience comme projet, comme temporalité qui se projette continuellement dans un horizon. À ce titre, le projet du collectif, toujours à venir précisément, est à animer et réanimer sans relâche. Il s'agit de le tenir presque à bout de bras - en mouvement par l'entremise d'une énergie commune, de le tenir en mouvance. L'ajout « à venir » est une manière d'insister sur le caractère inachevé et littéralement interminable du projet même du collectif. Cet ajout est aussi une manière de rappeler l'ouverture qui définit en propre le collectif : l'ouverture, dans les ateliers, à la déprise, à l'imprévisibilité, et ultimement à l'improvisation du "faire-avec» dont chacun fait l'épreuve. Rappelant l'enjeu politique de l'ouvrage, Patrick Chemla écrit dans sa conclusion :

Le Collectif à venir, [...] c'est aussi cette marche en avant au rythme que se fixe chacun, chaque collectif, pour affronter le temps et l'espace en refusant l'accélération folle du néolibéralisme, ou la marche en cadence militaire ou religieuse de la masse organisée (p. 244).

Ce passage offre finalement l'occasion d'interroger le collectif tel qu'il est convoqué dans le titre de l'ouvrage : le Collectif. Pourquoi reprendre le concept de collectif pour le réécrire ensuite ? Patrick Chemla recourt à une majuscule pour dépersonnaliser le concept, le relancer et le faire vivre au-delà de l'existence de Jean Oury. Parler du Collectif plutôt que du collectif est une autre façon encore d'indiquer le caractère interminable du projet du concept de Jean Oury. Toutefois, on peut se demander s'il n'eût pas été plus explicite de parler de « collectifs »? En effet, convoquer le collectif au pluriel permet, 
à la fois, de relancer le concept de Jean Oury et de rendre compte du fait, aussi essentiel que le caractère interminable du projet du collectif, que ce concept s'incarne dans des espace-temps situés - que l'on pense à La Borde et SaintAlban. Car dans les faits, derrière le collectif de Jean Oury et le Collectif de Patrick Chemla se cachent des collectifs particuliers : des collectifs qui se pensent dans un espace-temps spécifique, à partir de soigneux particuliers et d'activités propres.

Dès lors qu'elle a été mise au travail, la proposition d'Olivier Apprill selon laquelle le collectif n'existe qu'au niveau logique en tant que condition de possibilité - se prolonge comme suit : le collectif, en tant que concept qui se réfléchit inlassablement, est condition de possibilité de tous les collectifs concrets possibles. En cela, le collectif est proprement projet; il constitue à la fois le point de départ et l'horizon indépassable vers lequel les regards de chacun se tournent dans la pratique.

En fin de compte, Le Collectif à venir fait davantage pressentir ce que peut être le collectif qu'il en fournit une définition claire. Cet ouvrage s'apparente de manière concrète à un espace-temps qui permet aux auteurs de créer $\mathrm{du}$ commun. Explicite dès les premières lignes d'introduction et dans les dernières de conclusion sous la plume de Patrick Chemla, l'engagement politique des auteurs émaille l'ensemble du livre. De manière générale, une revendication dans le milieu des soins de santé est énoncée : il s'agit de lancer un mouvement de résistance contre la logique du néolibéralisme qui tend à inscrire l'individu dans de savants calculs de gestion.

Mais une question encore doit, au moins, être soulevée : à quel niveau situer en dernier ressort le propos du Collectif à venir? Y a-t-il une visée prescriptive derrière les expériences vécues décrites et rapportées par les auteurs ? Patrick Chemla a-t-il compilé un ensemble de recommandations qui indiquent implicitement, contre la loi de 2015 , ce qu'il faut faire en matière de soins de santé ? Déterminer le caractère descriptif et/ou prescriptif du recueil n'est pas évident. De nombreuses prises de parole semblent être descriptives en tant qu'elles rapportent des expériences singulières ; d'autres, par contre, paraissent davantage prescriptives, notamment en raison de leur positionnement explicite contre la loi de 2015. Ainsi Le Collectif à venir présente-t-il un caractère hybride. Il est à la fois descriptif et prescriptif : il dit ce qu'était le collectif chez Oury, il dit comment il parvient à se révéler présentement et il dit ce qu'il doit être demain pour échapper à la logique du néolibéralisme et avoir une chance d'exister encore.

Les mots de la fin sont laissés à Victoire Mabit. En tant que mère d'usager, cette dernière convoque la question de l'altérité. Elle tisse par-là un lien entre, d'une part, la démarche politique des auteurs et, d'autre part, un enjeu 
phénoménologique central du livre. Dans son texte intitulé « Le fil conducteur » (p. 161-163), elle insiste sur le rapport égalitaire qui doit régner entre soignants et soignés. Contre la réforme des GHT, elle énonce :

[J'écris] pour que les soignants résistent, désobéissent, et considèrent leurs patients comme des alter ego et non comme des sous-je-ne-sais-quoi qu'il faut tenir à carreau. [...] Je vois bien la pression, la compression que les directives de l'État mettent sur ceux qui veulent travailler humainement, avec leur conscience. J'ai envie de leur dire que la seule issue actuellement - je dis actuellement car la société évolue et rien n'est statique — c'est de résister. De désobéir. De pratiquer comme vous le voulez, comme vous le décidez, sans vous laisser détourner (p. 162).

On voit que le débat du collectif convoque l'importante question phénoménologique de l'altérité. Le propos de Victoire Mabit pose à ce sujet une série d'interrogations. Comment considère-t-on autrui ? Plus précisément, dans le cadre des soins en santé mentale : comment considère-t-on les personnes qui pensent le monde différemment? Les soignants sont-ils en droit de nier toute conscience aux individus qu'ils rencontrent? Peuvent-ils en faire des soussujets, des sous-je ? Derrière la question de l'altérité réside alors une question éminemment politique : existe-t-il des non-sujets à guérir ? Autrement dit, peut-on mettre entre parenthèses des subjectivités au motif de les réintégrer dans la société ? Peut-on considérer certains individus exclusivement à partir de leurs symptômes dans le seul but de les normaliser ? Serge Klopp ajoute, par ailleurs, un autre enjeu politique quand il écrit : «Cela parait idiot de dire que dans un hôpital le soin doit primer sur l'administratif, mais nous en sommes là » (p. 50). La question politique et polémique que pose finalement Le Collectif à venir à une société animée par une logique néolibérale peut être formulée comme suit : peut-on, dans une quelconque prise en charge, rayer le sujet et nier l'altérité au nom de la gestion administrative et/ou financière de l'institution? Dans la négative, il s'agit de poser - comme le fait Le Collectif à venir - la question phénoménologique suivante : comment appréhende-ton le sujet dans son rapport à soi, aux autres et au monde, dès lors qu'on le considère dans son individualité et comme un alter ego ? L'épreuve de l'altérité, explicitée par Victoire Mabit, constitue le nœud autour duquel s'entrecroisent les enjeux politiques et phénoménologiques du Collectif à venir. Entre son appel à la résistance voire à la désobéissance sur le plan politique et sa revendication à un rapport égalitaire entre soigneux, Le Collectif à venir offre à penser - en parallèle - des points fondamentaux de philosophie politique et de psychopathologie phénoménologique. 


\section{Notes}

1. «Réforme des GHT : quel bénéfice pour les usagers ? »URL : https://www.franceassos-sante.org/2016/07/08/reforme-des-ght-quel-benefice-pour-les-usagers/ (consulté le 5 décembre 2019).

2. «Réforme des GHT : quel bénéfice pour les usagers ?» (consulté le 7 décembre 2019).

3. France Culture : «Saint-Alban : lieu d'hospitalité 2/2. Une révolution psychiatrique », 24 novembre 2019. URL : https://www.franceculture.fr/emissions/ une-histoire-particuliere-un-recit-documentaire-en-deux-parties/saint-alban-lieudhospitalite-22-une-revolution-psychiatrique (consulté le 5 décembre 2019).

Géraldine Sauvage

(Université de Liège) 